MATHEMATICS OF COMPUTATION

Volume 71, Number 239, Pages 971-994

S 0025-5718(02)01412-6

Article electronically published on February 4, 2002

\title{
EACH AVERAGING TECHNIQUE YIELDS RELIABLE A POSTERIORI ERROR CONTROL IN FEM ON UNSTRUCTURED GRIDS. PART II: HIGHER ORDER FEM
}

\author{
SÖREN BARTELS AND CARSTEN CARSTENSEN
}

\begin{abstract}
Averaging techniques are popular tools in adaptive finite element methods since they provide efficient a posteriori error estimates by a simple postprocessing. In the second paper of our analysis of their reliability, we consider conforming $h$-FEM of higher (i.e., not of lowest) order in two or three space dimensions. In this paper, reliablility is shown for conforming higher order finite element methods in a model situation, the Laplace equation with mixed boundary conditions. Emphasis is on possibly unstructured grids, nonsmoothness of exact solutions, and a wide class of local averaging techniques. Theoretical and numerical evidence supports that the reliability is up to the smoothness of given right-hand sides.
\end{abstract}

\section{INTRODUCTION}

Given the exact solution $u$ and an approximate solution $u_{h}$ of a second order elliptic partial differential equation, an averaging technique for a posteriori error control is a postprocessing algorithm that provides $q_{h}$ from the input $\nabla u_{h}$. The true energy error $\left\|\nabla\left(u-u_{h}\right)\right\|_{L^{2}(\Omega)}$ is then estimated by $\left\|q_{h}-\nabla u_{h}\right\|_{L^{2}(\Omega)}$. The underlying motivation is that $q_{h}$ is a "smoother" approximation of $\nabla u$ or/and of "higher order". So far, mathematical justifications for regarding $\left\|q_{h}-\nabla u_{h}\right\|_{L^{2}(\Omega)}$ as an approximation to $\left\|\nabla\left(u-u_{h}\right)\right\|_{L^{2}(\Omega)}$ have been based on superconvergence phenomena or given for $\nabla u_{h}$ piecewise constant only. This paper aims to establish the reliability and efficiency of local averaging: For each edge $E$, written $E \in \mathcal{E}$, with neighbourhood $\omega_{E}$ (i.e., the union of elements with face $E$ ) we suggest to compute (in case of pure Dirichlet problems)

$$
\eta_{E}:=\min \left\{\left\|\nabla u_{h}-q_{E}\right\|_{L^{2}\left(\omega_{E}\right)}: q_{E} \in \mathcal{P}_{d_{E}}\left(\omega_{E}\right)^{d}\right\} \quad \text { and } \quad \eta_{Z}^{2}:=\sum_{E \in \mathcal{E}} \eta_{E}^{2} .
$$

The polynomial degree $d_{E}$ on $\omega_{E}$ is chosen according to the elementwise degrees of $u_{h}$ on $\omega_{E}$, and $\mathcal{P}_{d_{E}}\left(\omega_{E}\right)$ denotes algebraic polynomials of degree $\leq d_{E}$ regarded as functions on $\omega_{E}$. We analyse estimates of the form

$$
c_{1} \eta_{Z}+\text { h.o.t. } \leq\left\|\nabla\left(u-u_{h}\right)\right\|_{L^{2}(\Omega)} \leq c_{2} \eta_{Z}+\text { h.o.t. }
$$

Received by the editor February 17, 2000.

2000 Mathematics Subject Classification. Primary 65N30, 65R20, 74B20, 74G99, 74H99.

Key words and phrases. A posteriori error estimates, residual based error estimate, adaptive algorithm, reliability, finite element method, higher order finite element method. 
for the error estimator $\eta_{Z}=\left(\sum_{E \in \mathcal{E}} \eta_{E}^{2}\right)^{1 / 2}$ and study the qualitative behaviour of the constants $c_{1}, c_{2}>0$ and the higher order terms (h.o.t.) in (1.1). It turns out that the higher order terms in the efficiency estimate, i.e., the first inequality in (1.1), depend on the smoothness of the exact solution $u$ while the higher order terms in the reliability estimate, i.e., the second inequality in (1.1), merely depend on the smoothness of given right-hand sides. The constants $c_{1}$ and $c_{2}$ are independent of the mesh-size but depend on the polynomial degrees.

We stress that the upper bound of (1.1) shows reliability of any local averaging scheme in the sense that, whatever choice of $q_{E} \in \mathcal{P}_{d_{E}}\left(\omega_{E}\right)^{d}$ we have, up to higher order terms (which are easily computed from the given right-hand sides $f, g$, and $\left.u_{D}\right)$,

$$
\left\|\nabla\left(u-u_{h}\right)\right\|_{L^{2}(\Omega)} \leq c_{3}\left(\sum_{E \in \mathcal{E}}\left\|\nabla u_{h}-q_{E}\right\|_{L^{2}\left(\omega_{E}\right)}^{2}\right)^{1 / 2} .
$$

The error term $\eta_{E}$ measures the flux difference of two neighbouring elements and so might be regarded as an edge contribution generalising $h_{E} \int_{E}\left[\partial u_{h} / \partial n\right]^{2} d s$ from standard residual-based error estimations to higher order finite elements. Hence, (1.1) indicates the dominance of edge-contributions, which is true for lowest order finite elements $[\mathrm{CV}], \mathrm{R}$ but known to be false for general higher-order polynomials Y1, [Y2]. From this point of view, (1.2) appears surprising: the volume residual $f-\Delta_{\mathcal{T}} u_{h}$ does not enter the upper bound directly.

In case of lowest order finite elements, local and global averaging is indeed equivalent $\mathrm{CB}$, and so $q_{E}$ can be chosen as a $\mathcal{T}$-piecewise polynomial that is globally continuous, as suggested in [ZZ]. However, for higher order finite element methods it is conjectured that global averaging is not equivalent to local averaging.

The outline of the remaining part of this paper is as follows. Approximation and stability properties of a weak approximation operator of [Ca, [CV] are generalised to higher-order approximation in Section 2. Some preliminary results are given in Section 3 in the spirit of $[\mathrm{CB}]$. The main results on (1.1) are stated and proved in Section 4. Three examples with uniform, adapted, and perturbed meshes and a variety of polynomial order finite element methods conclude the paper in Section 5.

\section{Approximation in Higher order Finite ElEMENT SPACES}

The Lipschitz boundary $\Gamma=\partial \Omega$ of the bounded domain $\Omega$ is split into a closed Dirichlet part $\Gamma_{D}$ with positive surface measure and a remaining, relatively open and possibly empty, Neumann part $\Gamma_{N}:=\Gamma \backslash \Gamma_{D}$. Suppose $\mathcal{T}$ be a regular triangulation of the domain $\Omega \subseteq \mathbb{R}^{d}, d=1,2,3$, in the sense of Ciarlet [BS, Ci] (no hanging node, domain is matched exactly) with piecewise affine Lipschitz boundary $\Gamma=\partial \Omega=\Gamma_{D} \cup \Gamma_{N}$, i.e., $\mathcal{T}$ consists of a finite number of closed subsets of $\bar{\Omega}$, that cover $\bar{\Omega}=\bigcup \mathcal{T}$. Each element $T \in \mathcal{T}$ is either an interval $T=\operatorname{conv}\{a, b\}$ if $d=1$, a triangle $T=\operatorname{conv}\{a, b, c\}$ or a parallelogram $T=\operatorname{conv}\{a, b, c, d\}$ if $d=2$, and a tetrahedron $T=\operatorname{conv}\{a, b, c, d\}$ or a parallelepiped $T=\operatorname{conv}\{a, \ldots, h\}$ if $d=3$. The extremal points $a, b, c, d$ are called vertices; the faces $E \subseteq \partial T$, e.g. $E=\operatorname{conv}\{a, b\}$ in $d=2$ or $E=\operatorname{conv}\{a, b, c\}$ in $d=3$, are called edges. The set of all vertices and all edges appearing for some $T$ in $\mathcal{T}$ are denoted as $\mathcal{N}$ and $\mathcal{E}$. Two distinct and intersecting $T_{1}$ and $T_{2}$ share either an entire edge or a vertex. Each edge $E \in \mathcal{E}$ on the boundary $\Gamma$ belongs either to $\Gamma_{D}$, written $E \in \mathcal{E}_{D}$, or to $\bar{\Gamma}_{N}$, written 
$E \in \mathcal{E}_{N}$. Therefore the set of edges is partitioned into $\mathcal{E}_{\Omega}:=\{E \in \mathcal{E}: E \not \subset \Gamma\}, \mathcal{E}_{D}$, and $\mathcal{E}_{N}$. We stress that the union of all edges $\bigcup \mathcal{E}$ denotes the skeleton of edges in $\mathcal{T}$, i.e., the set of all points $x$ that belong to some boundary $x \in \partial T$ of some element $T \in \mathcal{T}$. Finally, $\mathcal{K}:=\mathcal{N} \backslash \Gamma_{D}$ denotes the set of free nodes.

For $T \in \mathcal{T}$, let $P_{T}^{k}:=\mathcal{P}_{k}(T)$ if $T$ is a triangle or tetrahedron, or $P_{T}^{k}:=\mathcal{Q}_{k}(T)$ if $T$ is a parallelogram or parallelepiped. Here, $\mathcal{P}_{k}(K)$ resp. $\mathcal{Q}_{k}(K)$ denotes the set of algebraic polynomials in $d$ variables on $K$ of total resp. partial degree $\leq k$. The space $\mathcal{L}^{k}(\mathcal{T})$ of (possibly discontinuous) $\mathcal{T}$-piecewise polynomials of degree $\leq k$ is the set of all $U \in L^{\infty}(\Omega)$ with $\left.U\right|_{T} \in P_{T}^{k}$ for all $T$ in $\mathcal{T}$. Set

$$
\mathcal{S}^{k}(\mathcal{T}):=\mathcal{L}^{k}(\mathcal{T}) \cap C(\Omega) \text { and } \mathcal{S}_{D}^{1}(\mathcal{T}):=\left\{u_{h} \in \mathcal{S}^{1}(\mathcal{T}):\left.u_{h}\right|_{\Gamma_{D}}=0\right\}
$$

Let $\left(\varphi_{z}: z \in \mathcal{N}\right)$ denote the nodal basis of $\mathcal{S}^{1}(\mathcal{T})$, i.e., $\varphi_{z} \in \mathcal{S}^{1}(\mathcal{T})$ satisfies $\varphi_{z}(x)=0$ if $x \in \mathcal{N} \backslash\{z\}$, and $\varphi_{z}(z)=1$. Note that $\left(\varphi_{z} \mid z \in \mathcal{N}\right)$ is a partition of unity and the open patches

$$
\omega_{z}:=\left\{x \in \Omega: 0<\varphi_{z}(x)\right\}
$$

form an open cover $\left(\omega_{z}: z \in \mathcal{N}\right)$ of $\Omega$ with finite overlap.

In order to define an approximation-operator $\mathcal{J}$, we choose for each fixed node $z \in \mathcal{N} \backslash \mathcal{K}$ a neighbouring free node $\zeta \in \mathcal{K}$ and thereby define a relation $R$ on $\mathcal{N}$ where $z R z$ if $z \in \mathcal{K}$. Then, let

$$
\psi_{z}:=\sum_{\zeta \in \mathcal{N}, \zeta R z} \varphi_{\zeta} \quad \text { and } \quad \Omega_{z}:=\operatorname{interior}\left(\operatorname{supp} \psi_{z}\right) .
$$

We require that for each $z \in \mathcal{K}, \Omega_{z}$ is connected and $\varphi_{z} \neq \psi_{z}$ implies that $\left(\partial \Omega_{z}\right) \cap \Gamma_{D}$ has a positive surface measure. As $(\{\zeta \in \mathcal{N}: \zeta R z\}: z \in \mathcal{K})$ is a partition of $\mathcal{N}$, $\left(\psi_{z}: z \in \mathcal{K}\right)$ is a partition of unity. For each $z \in \mathcal{K}$, we define the degree (minimal degree allowed on $\Omega_{z}$ minus one)

$$
d(z):=\max \left\{k \in \mathbb{N}_{0}: \mathcal{P}_{k}\left(\Omega_{z}\right) \varphi_{z} \subseteq \mathcal{S}\right\},
$$

where $\mathcal{P}_{k}\left(\Omega_{z}\right)$ denotes the set of all polynomials on $\mathbb{R}^{d}$ of total degree at most $k$ restricted to $\Omega_{z}$. The set $\mathcal{S} \subseteq H^{1}(\Omega)$ is some finite element space consisting of functions that are $\mathcal{T}$-elementwise polynomials and globally continuous. Moreover, we require that $\mathcal{S}_{D}^{1}(\mathcal{T}) \subseteq \mathcal{S}$, which implies that $d(z)$ is well defined and greater than or equal than zero.

For $g \in L^{1}(\Omega)$ and $z \in \mathcal{K}$, let $g_{z} \in \mathcal{P}_{d(z)}\left(\Omega_{z}\right)$ be defined by

$$
\int_{\Omega_{z}}\left(g_{z} \varphi_{z}-g \psi_{z}\right) q_{z} d x=0 \text { for all } q_{z} \in \mathcal{P}_{d(z)}\left(\Omega_{z}\right),
$$

and then let

$$
\mathcal{J} g:=\sum_{z \in \mathcal{K}} g_{z} \varphi_{z} \in \mathcal{S} \cap H_{D}^{1}(\Omega) .
$$

Remark 2.1. Notice that $g_{z} \varphi_{z} \in \mathcal{S}$, because of (2.3).

Remark 2.2. Condition (2.4) characterises the unique minimiser $g_{z}$ of

$$
\frac{1}{2} \int_{\Omega_{z}} \varphi_{z} q_{z}^{2} d x-\int_{\Omega_{z}} g \psi_{z} q_{z} d x
$$

amongst all $q_{z}$ in $\mathcal{P}_{d(z)}\left(\Omega_{z}\right)$. In particular, there exists a unique solution to (2.4), i.e., $\mathcal{J} g$ is well defined and belongs to $\mathcal{S}$ even for $g \in L^{1}(\Omega)$. 
The local mesh-sizes are denoted by $h_{\mathcal{T}}$ and $h_{\mathcal{E}}$, where $h_{\mathcal{T}} \in \mathcal{L}^{0}(\mathcal{T})$ is the elementsize, $\left.h_{\mathcal{T}}\right|_{T}:=h_{T}:=\operatorname{diam}(T)$ for $T \in \mathcal{T}$, and the edge-size $h_{\mathcal{E}} \in L^{\infty}(\bigcup \mathcal{E})$ is defined on the union or skeleton $\bigcup \mathcal{E}$ of all edges $E$ in $\mathcal{E}$ by $\left.h_{\mathcal{E}}\right|_{E}:=h_{E}:=\operatorname{diam}(E)$. The patch-size $h_{z}:=\operatorname{diam}\left(\Omega_{z}\right)$ is defined for each node $z \in \mathcal{K}$ separately.

Theorem 2.1. There exist $\left(h_{\mathcal{T}}, h_{\mathcal{E}}\right)$-independent constants $c_{4}, c_{5}, c_{6}, c_{7}>0$ such that, for all $g \in H_{D}^{1}(\Omega)$ and $f \in L^{2}(\Omega)$,

$$
\begin{aligned}
\|\nabla(g-\mathcal{J} g)\|_{L^{2}(\Omega)} & \leq c_{4}\|\nabla g\|_{L^{2}(\Omega)} \\
\int_{\Omega} f(g-\mathcal{J} g) d x \leq & c_{5}\|\nabla g\|_{L^{2}(\Omega)} \\
& \times\left(\sum_{z \in \mathcal{K}} h_{z}^{2} \min _{f_{z} \in \mathcal{P}_{d(z)}\left(\Omega_{z}\right)}\left\|f-f_{z}\right\|_{L^{2}\left(\Omega_{z}\right)}^{2}\right)^{1 / 2}, \\
\left\|h_{\mathcal{T}}^{-1}(g-\mathcal{J} g)\right\|_{L^{2}(\Omega)} \leq & c_{6}\|\nabla g\|_{L^{2}(\Omega)}, \\
\left\|h_{\mathcal{E}}^{-1 / 2}(g-\mathcal{J} g)\right\|_{L^{2}\left(\Gamma_{N}\right)} & \leq c_{7}\|\nabla g\|_{L^{2}(\Omega)} .
\end{aligned}
$$

The constants $c_{4}, c_{5}, c_{6}, c_{7}$ only depend on $\Omega, \Gamma_{D}, \Gamma_{N}$, the degrees $d(z), z \in \mathcal{K}$, and the shapes of the elements $T \in \mathcal{T}$ and the patches $\Omega_{z}, z \in \mathcal{K}$.

Proof. In this proof and at similar occasions, $\lesssim$ abbreviates an inequality $\leq$ up to a constant $\left(h_{\mathcal{T}}, h_{\mathcal{E}}\right)$-independent factor. Also, $\|\cdot\|_{p, K}$ abbreviates $\|\cdot\|_{L^{p}(K)}$ and we neglect $K$ if $\Omega$ is meant, i.e., $\|\cdot\|_{2}:=\|\cdot\|_{2, \Omega}$. Hence, e.g., (2.7) could be phrased as $\|\nabla \mathcal{J} g-\nabla g\|_{2} \lesssim\|\nabla g\|_{2}$.

The local key estimate for the stability and the approximation property of $\mathcal{J}$ will be

$$
\left\|g_{z} \varphi_{z}-g \psi_{z}\right\|_{2, \Omega_{z}} \lesssim h_{z}\|\nabla g\|_{2, \Omega_{z}} .
$$

(The constant in (2.11) is $h_{z}$-independent but depends on $d(z)$ and the shape of $\Omega_{z}$.) For the proof of (2.11) let $\bar{g}_{z}$ denote the integral mean of $g$ on $\Omega_{z}$. Then, $g_{z}-\bar{g}_{z} \in \mathcal{P}_{d(z)}\left(\Omega_{z}\right)$ and

$$
\left\|g_{z}-\bar{g}_{z}\right\|_{2, \Omega_{z}} \leq c_{8}\left\|\varphi_{z}\left(g_{z}-\bar{g}_{z}\right)\right\|_{2, \Omega_{z}} \text {. }
$$

For a proof of (2.12), notice that $\|\cdot\|_{2, \Omega_{z}}$ and $\left\|\varphi_{z} \cdot\right\|_{2, \Omega_{z}}$ are norms on $\mathcal{P}_{d(z)}\left(\Omega_{z}\right)$ and so are equivalent. A scaling argument shows that the constant $c_{8}$ is $h_{z}$-independent (but of course depends on the shape of $\Omega_{z}$ and the degree $d(z)$ ). Since $\bar{g}_{z} \in$ $\mathcal{P}_{d(z)}\left(\Omega_{z}\right)$ is allowed in (2.6), we have

$$
\int_{\Omega_{z}} \varphi_{z} g_{z}^{2} d x \leq \int_{\Omega_{z}} \varphi_{z} \bar{g}_{z}^{2} d x-2 \int_{\Omega_{z}} \psi_{z} g \bar{g}_{z} d x+2 \int_{\Omega_{z}} \psi_{z} g g_{z} d x
$$


and infer, with Cauchy's and Young's inequality,

$$
\begin{aligned}
\|\left(g_{z}-\right. & \left.\bar{g}_{z}\right) \varphi_{z}\left\|_{2, \Omega_{z}}^{2} \leq\right\|\left(g_{z}-\bar{g}_{z}\right) \varphi_{z}^{1 / 2} \|_{2, \Omega_{z}}^{2} \\
= & 2 \int_{\Omega_{z}} \varphi_{z} \bar{g}_{z}\left(\bar{g}_{z}-g_{z}\right) d x+2 \int_{\Omega_{z}} \psi_{z} g\left(g_{z}-\bar{g}_{z}\right) d x \\
= & 2 \int_{\Omega_{z}} \varphi_{z}\left(\bar{g}_{z}-g\right)\left(\bar{g}_{z}-g_{z}\right) d x+2 \int_{\Omega_{z}}\left(\psi_{z}-\varphi_{z}\right) g\left(g_{z}-\bar{g}_{z}\right) d x \\
\leq & \frac{1}{4}\left\|\left(g_{z}-\bar{g}_{z}\right) \varphi_{z}\right\|_{2, \Omega_{z}}^{2}+4\left\|g-\bar{g}_{z}\right\|_{2, \Omega_{z}}^{2} \\
& +\frac{1}{4 c_{8}^{2}}\left\|g_{z}-\bar{g}_{z}\right\|_{2, \Omega_{z}}^{2}+4 c_{8}^{2}\left\|\left(\psi_{z}-\varphi_{z}\right) g\right\|_{2, \Omega_{z}}^{2} .
\end{aligned}
$$

Utilising (2.12) and absorbing $\left\|\left(g_{z}-\bar{g}_{z}\right) \varphi_{z}\right\|_{2, \Omega_{z}}^{2}$, we deduce from (2.14) that

$$
\left\|\left(g_{z}-\bar{g}_{z}\right) \varphi_{z}\right\|_{2, \Omega_{z}}^{2} \leq 8\left\|g-\bar{g}_{z}\right\|_{2, \Omega_{z}}^{2}+8 c_{8}^{2}\left\|\left(\psi_{z}-\varphi_{z}\right) g\right\|_{2, \Omega_{z}}^{2} .
$$

A Poincaré inequality yields

$$
\left\|g-\bar{g}_{z}\right\|_{2, \Omega_{z}} \lesssim h_{z}\|\nabla g\|_{2, \Omega_{z}}
$$

with a constant factor that only depends on the shape of $\Omega_{z}$; the weight-function $\left(\psi_{z}-\varphi_{z}\right)$ is non-zero only if $\Gamma_{D} \cap\left(\partial \Omega_{z}\right)$ has positive surface measure. Since $g=0$ there, a Friedrichs' inequality yields

$$
\left\|\left(\psi_{z}-\varphi_{z}\right) g\right\|_{2, \Omega_{z}} \leq\|g\|_{2, \Omega_{z}} \lesssim h_{z}\|\nabla g\|_{2, \Omega_{z}}
$$

for an $h_{z}$-independent constant that depends on the shape of $\Omega_{z}$ and $\left(\partial \Omega_{z}\right) \cap \Gamma_{D}$ only. Therefore, (2.15) yields

$$
\left\|\left(g_{z}-\bar{g}_{z}\right) \varphi_{z}\right\|_{2, \Omega_{z}} \lesssim h_{z}\|\nabla g\|_{2, \Omega_{z}} .
$$

To prove (2.11), we use the triangle inequality and (2.15)-(2.18) to verify

$$
\begin{aligned}
\left\|g_{z} \varphi_{z}-g \psi_{z}\right\|_{2, \Omega_{z}} \leq\left\|\left(g_{z}-\bar{g}_{z}\right) \varphi_{z}\right\|_{2, \Omega_{z}} & +\left\|\left(g-\bar{g}_{z}\right) \varphi_{z}\right\|_{2, \Omega_{z}} \\
& +\left\|\left(\psi_{z}-\varphi_{z}\right) g\right\|_{2, \Omega_{z}} \lesssim h_{z}\|\nabla g\|_{2, \Omega_{z}},
\end{aligned}
$$

which is (2.11). To verify (2.8), we use that $\left(\psi_{z}: z \in \mathcal{K}\right)$ is a partition of unity and obtain with (2.11), (2.4) for any $f_{z} \in \mathcal{P}_{d(z)}\left(\Omega_{z}\right)$ that

$$
\begin{gathered}
\int_{\Omega} f(g-\mathcal{J} g) d x=\sum_{z \in \mathcal{K}} \int_{\Omega_{z}} f\left(g \psi_{z}-g_{z} \varphi_{z}\right) d x=\sum_{z \in \mathcal{K}} \int_{\Omega_{z}}\left(f-f_{z}\right)\left(g \psi_{z}-g_{z} \varphi_{z}\right) d x \\
\lesssim \sum_{z \in \mathcal{K}}\left\|f-f_{z}\right\|_{2, \Omega_{z}} h_{z}\|\nabla g\|_{2, \Omega_{z}} \lesssim\left(\sum_{z \in \mathcal{K}} h_{z}^{2}\left\|f-f_{z}\right\|_{2, \Omega_{z}}^{2}\right)^{1 / 2}\|\nabla g\|_{2} .
\end{gathered}
$$

In the last step we used that $\left(\psi_{z}: z \in \mathcal{K}\right)$ has a finite overlap that depends on the shape of the elements only. The proof of (2.8) is finished. Notice that $h_{z} \lesssim h_{T}$ for all $z \in \mathcal{K}$ and $T \in \mathcal{T}$ with $T \subseteq \bar{\Omega}_{z}$. Letting $f:=h_{\mathcal{T}}^{-2}(g-\mathcal{J} g)$ and $f_{z}=0, z \in \mathcal{K}$, we deduce from (2.8) that

$$
\begin{aligned}
\left\|h_{\mathcal{T}}^{-1}(g-\mathcal{J} g)\right\|_{2}^{2} & \lesssim\|\nabla g\|_{2}\left(\sum_{z \in \mathcal{K}}\left\|h_{\mathcal{T}}^{-1}(g-\mathcal{J} g)\right\|_{2, \Omega_{z}}^{2}\right)^{1 / 2} \\
& \lesssim\|\nabla g\|_{2}\left\|h_{\mathcal{T}}^{-1}(g-\mathcal{J} g)\right\|_{2}
\end{aligned}
$$


which implies (2.9)). To verify (2.7) we argue as above and additionally utilise $\sum_{z \in \mathcal{K}} \nabla \psi_{z}=0$. Repeating the triangle inequality only a limited number of times (according to the finite overlap of the patches $\Omega_{z}$ ), we infer

$$
\|\nabla g-\nabla \mathcal{J} g\|_{2}^{2} \lesssim \sum_{z \in \mathcal{K}}\left\|\nabla\left(\psi_{z} g-\varphi_{z} g_{z}\right)\right\|_{2}^{2} .
$$

Since $\bar{g}_{z}$ is constant, and recalling (2.16), we have

$$
h_{z}^{-1}\left\|g-\bar{g}_{z}\right\|_{2, \Omega_{z}}+\left\|\nabla\left(g-\bar{g}_{z}\right)\right\|_{2, \Omega_{z}} \lesssim\|\nabla g\|_{2, \Omega_{z}} .
$$

Then, with the triangle inequality, Friedrichs' inequality, $\left\|\nabla \phi_{\zeta}\right\|_{\infty, \Omega_{z}} \leq 1 / \rho_{z}$, and (2.23) we conclude that

$$
\begin{aligned}
& \left\|\nabla\left(g \psi_{z}-g_{z} \varphi_{z}\right)\right\|_{2, \Omega_{z}} \\
& \quad \leq\left\|\left(\psi_{z}-\varphi_{z}\right) \nabla g\right\|_{2, \Omega_{z}}+\left\|\nabla\left(\varphi_{z}\left(g_{z}-\bar{g}_{z}\right)\right)\right\|_{2, \Omega_{z}} \\
& \quad+\left\|\nabla\left(\varphi_{z}\left(\bar{g}_{z}-g\right)\right)\right\|_{2, \Omega_{z}}+\left\|g \nabla\left(\psi_{z}-\varphi_{z}\right)\right\|_{2, \Omega_{z}} \\
& \quad \lesssim\|\nabla g\|_{2, \Omega_{z}}+h_{z} / \rho_{z}\|\nabla g\|_{2, \Omega_{z}}+\left\|\nabla\left(\varphi_{z}\left(g_{z}-\bar{g}_{z}\right)\right)\right\|_{2, \Omega_{z}} .
\end{aligned}
$$

Note that $h_{z} \lesssim \rho_{z}$. To estimate $\left\|\nabla\left(\varphi_{z}\left(g_{z}-\bar{g}_{z}\right)\right)\right\|_{2, \Omega_{z}}$ we observe that $\left\|\nabla\left(\varphi_{z} \cdot\right)\right\|_{2, \Omega_{z}}$ is a norm on $\mathcal{P}_{d(z)}\left(\Omega_{z}\right)$ and so is equivalent to $\left\|\varphi_{z} \cdot\right\|_{2, \Omega_{z}}$. A scaling argument shows that

$$
h_{z}\left\|\nabla\left(\varphi_{z}\left(g_{z}-\bar{g}_{z}\right)\right)\right\|_{2, \Omega_{z}} \lesssim\left\|\varphi_{z}\left(g_{z}-\bar{g}_{z}\right)\right\|_{2, \Omega_{z}},
$$

where the $h_{z}$-independent constant depends on the shape of $\Omega_{z}$ and the degree $d(z)$ only. Utilising (2.11), (2.23), and Friedrichs' inequality, we obtain from (2.25) that

$$
\begin{aligned}
\frac{h_{z}}{c_{12}}\left\|\nabla\left(\varphi_{z}\left(g_{z}-\bar{g}_{z}\right)\right)\right\|_{2, \Omega_{z}} \leq & \left\|\varphi_{z} g_{z}-\psi_{z} g\right\|_{2, \Omega_{z}} \\
& +\left\|\left(\psi_{z}-\varphi_{z}\right) g\right\|_{2, \Omega_{z}}+\left\|\varphi_{z}\left(g-\bar{g}_{z}\right)\right\|_{2, \Omega_{z}} \\
\lesssim & h_{z}\|\nabla g\|_{2, \Omega_{z}} .
\end{aligned}
$$

Employing this estimate in (2.24) and the resulting estimate in (2.22), we verify (2.7).

A trace inequality $[\mathrm{BS}], \overline{\mathrm{Cl}}], \overline{\mathrm{CF}}$ is required for the proof of (2.10). For $E \in \mathcal{E}_{N}$ and a neighbouring element $T \in \mathcal{T}$ with $E \subset \partial T \cap \bar{\Gamma}_{N}$ we have, for all $w \in H^{1}(T)$,

$$
\|w\|_{2, E} \lesssim h_{E}^{-1 / 2}\|w\|_{2, T}+h_{E}^{1 / 2}\|\nabla w\|_{2, T} .
$$

We denote $\omega_{E}:=T$ for $E \in \mathcal{E}_{N}$ and $E \subseteq \partial T$. Since $h_{T} \lesssim h_{E}$, we deduce from (2.26) with $w=g-\mathcal{J} g$ by summing all edges $E$ on $\Gamma_{N}$ that

$$
\begin{aligned}
\sum_{E \in \mathcal{E}_{N}} h_{E}^{-1}\|g-\mathcal{J} g\|_{2, E}^{2} & \lesssim \sum_{E \in \mathcal{E}_{N}} h_{T}^{-2}\|g-\mathcal{J} g\|_{2, \omega_{E}}^{2}+\sum_{E \in \mathcal{E}_{N}}\|\nabla(g-\mathcal{J} g)\|_{2, \omega_{E}}^{2} \\
& \lesssim\|\nabla g\|_{2, \Omega}^{2},
\end{aligned}
$$

according to (2.7) and (2.9) . This concludes the proof.

Remark 2.3. The constants in the theorem depend on the polynomial degrees in the finite element method and are expected to increase to infinity with the degree. 


\section{BASIC ESTIMATES}

In this section we first derive with the approximation operator $\mathcal{J}$ a global error estimate for a posteriori error control by averaging processes in an abstract setting. The estimate of this section is specified in the subsequent section to higher order conforming finite element methods.

Theorem 3.1. Suppose $p, q \in H(\operatorname{div} ; \Omega)$ and $p_{h} \in \mathcal{L}^{k}(\mathcal{T})^{d}$ with $p \cdot n, q \cdot n \in L^{2}\left(\Gamma_{N}\right)$ and

$$
\int_{\Omega}\left(p-p_{h}\right) \cdot \nabla w_{h} d x=0 \quad \text { for all } w_{h} \in \mathcal{S} \cap H_{D}^{1}(\Omega)
$$

Then

$$
\begin{aligned}
& \sup _{\substack{w \in H_{D}^{1}(\Omega) \\
\|\nabla w\|_{L^{2}(\Omega)}=1}} \int_{\Omega}\left(p-p_{h}\right) \cdot \nabla w d x \leq c_{4}\left\|p_{h}-q\right\|_{L^{2}(\Omega)} \\
& \left.\quad+c_{5}\left(\sum_{z \in \mathcal{K}} h_{z}^{2} \min _{f_{z} \in \mathcal{P}_{d(z)}\left(\Omega_{z}\right)}\left\|\operatorname{div}(p-q)-f_{z}\right\|_{L^{2}\left(\Omega_{z}\right)}\right)^{2}\right)^{1 / 2} \\
& \quad+c_{7}\left\|h_{\mathcal{E}}^{1 / 2}(p-q) \cdot n\right\|_{L^{2}\left(\Gamma_{N}\right) .}
\end{aligned}
$$

Proof. According to (3.1), (2.7), Cauchy's inequality, and an integration by parts we have, for each $w \in H_{D}^{1}(\Omega)$ with $\|\nabla w\|_{2}=1$, that

$$
\begin{aligned}
\int_{\Omega}\left(p-p_{h}\right) \cdot \nabla w d x=\int_{\Omega}\left(p-p_{h}\right) \cdot \nabla(w-\mathcal{J} w) d x \\
=\int_{\Omega}(p-q) \cdot \nabla(w-\mathcal{J} w) d x+\int_{\Omega}\left(q-p_{h}\right) \cdot \nabla(w-\mathcal{J} w) d x \\
\leq \int_{\Gamma_{N}}(w-\mathcal{J} w)(p-q) \cdot n d x \\
\quad-\int_{\Omega}(w-\mathcal{J} w) \operatorname{div}(p-q) d x+c_{4}\left\|p_{h}-q\right\|_{2, \Omega},
\end{aligned}
$$

since $w$ and $\mathcal{J} w$ vanish on $\partial \Omega \backslash \Gamma_{N}$. Owing to (2.8) and (2.10) in Theorem 2.1, we conclude (3.2) from (3.3) and Cauchy's inequality.

The next lemma states that averaging over the patches $\Omega_{z}$ is dominated by averaging over smaller domains. Let $\omega_{E}:=T_{1} \cup T_{2}$ for an interior edge $E=$ $T_{1} \cap T_{2} \in \mathcal{E}_{\Omega}$ and $\omega_{E}:=T$ for an edge $E=T \cap \partial \Omega \in \mathcal{E}_{D} \cup \mathcal{E}_{N}$ on the boundary. Moreover, for $z \in \mathcal{K}$, let $\mathcal{E}_{\Omega_{z}}$ denote the set of all edges $E \subset \bar{\Omega}_{z}$ with $E \not \subset \partial \Omega_{z}$.

Lemma 3.1. Suppose $\mathcal{S}=\left\{v_{h} \in C(\bar{\Omega}): \forall T \in \mathcal{T},\left.v_{h}\right|_{T} \in \mathcal{P}_{d(T)}(T)\right\}$ for positive integers $d(T), T \in \mathcal{T}$, and let $d_{E}, E \in \mathcal{E}$, be nonnegative integers. Then there exists a constant $c_{9}>0$ such that, for all $u_{h} \in \mathcal{S}$ and each $z \in \mathcal{K}$, we have

$$
\min _{q_{z} \in \mathcal{P}_{d(z)+1}\left(\Omega_{z}\right)^{d}}\left\|\nabla u_{h}-q_{z}\right\|_{L^{2}\left(\Omega_{z}\right)}^{2} \leq c_{9} \sum_{E \in \mathcal{E}_{\Omega_{z}}} \min _{q_{E} \in \mathcal{P}_{d_{E}}\left(\omega_{E}\right)^{d}}\left\|\nabla u_{h}-q_{E}\right\|_{L^{2}\left(\omega_{E}\right)}^{2} .
$$

The constant $c_{9}$ depends on the degrees $d_{z}$ and $d_{E}$ as well as on the shapes of the elements and patches, but not on their diameters.

Proof. Set $p_{h}:=\nabla u_{h}$ and let $\left\|\left|p_{h}\right|\right\|_{1, z}^{2}$ and $\left\|\left|p_{h}\right|\right\|_{2, z}^{2}$ denote the expressions on the left- and right-hand side in (3.4), respectively. Assume $u_{h} \in \mathcal{S}$ with $\left\|\left|p_{h}\right|\right\|_{2, z}=0$. 
We claim that $\left\|\left|p_{h}\right|\right\|_{1, z}=0$. (The statement of the lemma then follows from compactness and scaling argument.) Since $\Omega_{z}$ is connected, there exists a sequence of edges $E_{1}, \ldots, E_{J} \in \mathcal{E}_{\Omega_{z}}$ such that $\omega_{E_{j}} \cap \omega_{E_{j+1}} \neq \emptyset$ and the open patches $\omega_{E_{j}}$ cover $\Omega_{z}$, i.e., $\omega_{E_{1}} \cup \ldots \cup \omega_{E_{J}}=\Omega_{z}$. Since $\left\|\left|p_{h}\right|\right\|_{2, z}=0$ we deduce that $\left.p_{h}\right|_{\omega_{E_{j}}} \in \mathcal{P}_{d_{E_{j}}}\left(\omega_{E_{j}}\right)^{d}$, $j=1, \ldots, J$, and so $\left.p_{h}\right|_{\Omega_{z}} \in \mathcal{P}_{m}\left(\Omega_{z}\right)^{d}$, where $m$ is the smallest polynomial degree of $p_{h}$ restricted to elements $T \subseteq \bar{\Omega}_{z}$, i.e., $m=\min \left\{m_{T}: T \subseteq \bar{\Omega}_{z}\right\}$ for $m_{T}:=\min \left\{\ell \in \mathbb{N}_{0}:\left.p_{h}\right|_{T} \in \mathcal{P}_{\ell}(T)^{d}\right\}$. Since $m \leq d(T)-1$ for all $T \subseteq \bar{\Omega}_{z}$ and $d(z) \geq \min _{T \in \mathcal{T}, T \subseteq \bar{\Omega}_{z}} d(T)-2$, we have $m \leq d(z)+1$. This implies $\left\|\left|p_{h}\right|\right\|_{1, z}=0$.

Remark 3.1. Note that a hat function $\varphi_{z}$ is not elementwise affine when $z$ belongs to a parallelogram if $d=2$ or a parallelepiped if $d=3$. Then, $\mathcal{P}_{d(z)+1}\left(\Omega_{z}\right)^{d}$ cannot be reduced to $\mathcal{P}_{d(z)}\left(\Omega_{z}\right)^{d}$ in (3.4).

The following lemma includes the approximation of given boundary data by discrete functions. We denote by $\mathcal{L}^{k}\left(\mathcal{E}_{N}\right)$ the space of all (possibly discontinuous) functions on $\Gamma_{N}$ which equal a polynomial of degree at most $k$ on each edge $E \in \mathcal{E}_{N}$.

Lemma 3.2. Let $k$ and $d_{E}, E \in \mathcal{E}$, be nonnegative integers and let $p_{h} \in \mathcal{L}^{k}(\mathcal{T})^{d}$ and $g_{h} \in \mathcal{L}^{k+1}\left(\mathcal{E}_{N}\right)$. Then,

$$
\begin{aligned}
& \min _{q_{h} \in \mathcal{S}^{k+1}(\mathcal{T})^{d}}\left(\left\|p_{h}-q_{h}\right\|_{L^{2}(\Omega)}^{2}+\left\|h_{\mathcal{E}}^{1 / 2}\left(g_{h}-q_{h} \cdot n\right)\right\|_{L^{2}\left(\Gamma_{N}\right)}^{2}\right) \\
& \quad \leq c_{10} \sum_{E \in \mathcal{E}_{\Omega} \cup \mathcal{E}_{N}} \min _{q_{E} \in \mathcal{P}_{d_{E}}\left(\omega_{E}\right)^{d}}\left(\left\|p_{h}-q_{E}\right\|_{L^{2}\left(\omega_{E}\right)}^{2}+h_{E}\left\|g_{h}-q_{E} \cdot n\right\|_{L^{2}\left(E \cap \Gamma_{N}\right)}^{2}\right)
\end{aligned}
$$

with a constant $c_{10}>0$ that depends on the degrees $k$ and $d_{E}$ as well as on the shapes of the elements and patches but not on their diameters.

Proof. Let $\mathcal{T}_{E}:=\left\{T \in \mathcal{T}: T \subseteq \bar{\omega}_{E}\right\}$ denote the restriction of $\mathcal{T}$ to $\omega_{E}, E \in \mathcal{E}$. It is shown in $\mathrm{CB}$ that

$$
\begin{aligned}
& \min _{q_{h} \in \mathcal{S}^{k+1}(\mathcal{T})^{d}}\left(\left\|p_{h}-q_{h}\right\|_{2, \Omega}^{2}+\left\|h_{\mathcal{E}}^{1 / 2}\left(g_{h}-q_{h} \cdot n\right)\right\|_{2, \Gamma_{N}}^{2}\right) \\
& \quad \lesssim \sum_{E \in \mathcal{E}_{\Omega} \cup \mathcal{E}_{N}} \min _{q_{E} \in \mathcal{S}^{k+1}\left(\mathcal{T}_{E}\right)^{d}}\left(\left\|p_{h}-q_{E}\right\|_{2, \omega_{E}}^{2}+h_{E}\left\|g_{h}-q_{E} \cdot n\right\|_{2, E \cap \Gamma_{N}}^{2}\right) .
\end{aligned}
$$

For $E \in \mathcal{E}_{\Omega}$ we have $\min _{q_{E} \in \mathcal{P}_{d_{E}}\left(\omega_{E}\right)^{d}}\left\|p_{h}-q_{E}\right\|_{2, \omega_{E}}^{2}=0$ if and only if $p_{h} \in$ $\mathcal{P}_{d_{E}}\left(\omega_{E}\right)^{d} \cap \mathcal{L}^{k}\left(\mathcal{T}_{E}\right)^{d} \subset C\left(\omega_{E}\right)^{d}$, and $\min _{q_{E} \in \mathcal{S}^{k+1}\left(\mathcal{T}_{E}\right)^{d}}\left\|p_{h}-q_{E}\right\|_{2, \omega_{E}}^{2}=0$ if and only if $p_{h} \in C\left(\omega_{E}\right)^{d} \cap \mathcal{L}^{k}\left(\mathcal{T}_{E}\right)^{d}$. Hence a compactness and a scaling argument (from the context of equivalence of norms) show (with an $\left(h_{\mathcal{T}}, h_{\mathcal{E}}\right)$-independent constant factor)

$$
\min _{q_{E} \in \mathcal{S}^{k+1}\left(\mathcal{T}_{E}\right)^{d}}\left\|p_{h}-q_{E}\right\|_{2, \omega_{E}}^{2} \lesssim \min _{q_{E} \in \mathcal{P}_{d_{E}}\left(\omega_{E}\right)^{d}}\left\|p_{h}-q_{E}\right\|_{2, \omega_{E}}^{2} .
$$

For an edge $E \in \mathcal{E}_{N}$ the corresponding minimum in the right-hand-side of (B.6) is zero if and only if $g_{h}=p_{h} \cdot n$, while the corresponding minimum over $\mathcal{P}_{d_{E}}\left(\omega_{E}\right)^{d}$ is zero if and only if $g_{h}=p_{h} \cdot n$ and $\left.p_{h}\right|_{\omega_{E}} \in \mathcal{P}_{d_{E}}\left(\omega_{E}\right)^{d}$. By a compactness and a 
scaling argument we deduce the inequality

$$
\begin{aligned}
\min _{q_{E} \in \mathcal{S}^{k+1}\left(\mathcal{T}_{E}\right)^{d}}\left(\left\|p_{h}-q_{E}\right\|_{2, \omega_{E}}^{2}+h_{E}\left\|g_{h}-q_{E} \cdot n\right\|_{2, E \cap \Gamma_{N}}^{2}\right) & \\
& \lesssim \min _{q_{E} \in \mathcal{P}_{d_{E}}\left(\omega_{E}\right)^{d}}\left(\left\|p_{h}-q_{E}\right\|_{2, \omega_{E}}^{2}+h_{E}\left\|g_{h}-q_{E} \cdot n\right\|_{2, E \cap \Gamma_{N}}^{2}\right) .
\end{aligned}
$$

Combining (3.6), (3.7), and (3.8), we obtain (3.5).

\section{Higher ORDER Finite ELEMENT METHOdS}

Given right-hand sides $f \in L^{2}(\Omega), g \in L^{2}\left(\Gamma_{N}\right)$, and $u_{D} \in H^{1}\left(\Gamma_{D}\right) \cap C\left(\Gamma_{D}\right)$, let $u \in H^{1}(\Omega)$ denote the unique weak solution to

$$
\begin{array}{rlrl}
-\Delta u & =f & \text { in } \Omega, \\
u & =u_{D} & \text { on } \Gamma_{D}, \\
\partial u / \partial n & =g & & \text { on } \Gamma_{N} .
\end{array}
$$

Suppose a finite element scheme, based on a regular triangulation $\mathcal{T}$, provided a discrete flux $p_{h}:=\nabla u_{h}$ to the exact flux $p:=\nabla u \in H(\operatorname{div} ; \Omega)$ such that $u_{h} \in \mathcal{S}$ and

$$
\int_{\Omega} \nabla u_{h} \cdot \nabla w_{h} d x=\int_{\Omega} f w_{h} d x+\int_{\Gamma_{N}} g w_{h} d s \quad \text { for all } w_{h} \in \mathcal{S} \cap H_{D}^{1}(\Omega) .
$$

We will assume in Lemma 4.3 below that $\left.u_{h}\right|_{\Gamma_{D}}$ interpolates $u_{D}$ in nodes on $\Gamma_{D}$.

Theorem 4.1. Assume $\nabla u_{h} \in \mathcal{L}^{k}(\mathcal{T})^{d}$ and (4.4). There exist $\left(h_{\mathcal{T}}, h_{\mathcal{E}}\right)$-independent constants $c_{11}, c_{12}>0$ such that

$$
\begin{aligned}
\| \nabla(u- & \left.u_{h}\right) \|_{L^{2}(\Omega)} \\
\leq & \min _{q_{h} \in \mathcal{S}^{k+1}(\mathcal{T})^{d}}\left(c_{11}\left\|\nabla u_{h}-q_{h}\right\|_{L^{2}(\Omega)}+2 c_{7}\left\|h_{\mathcal{E}}^{1 / 2}\left(g-q_{h} \cdot n\right)\right\|_{L^{2}\left(\Gamma_{N}\right)}\right) \\
& +c_{12}\left(\sum_{z \in \mathcal{K}^{\prime}} \min _{q_{z} \in \mathcal{P}_{d(z)+1}\left(\Omega_{z}\right)^{d}}\left\|\nabla u_{h}-q_{z}\right\|_{L^{2}\left(\Omega_{z}\right)}^{2}\right)^{1 / 2} \\
& +\inf _{\left.v\right|_{\Gamma_{D}}=u_{D}}\left\|\nabla\left(u_{h}-v\right)\right\|_{L^{2}(\Omega)} \\
& +2 \sqrt{3} c_{5}\left(\sum_{z \in \mathcal{K}} h_{z}^{2} \min _{f_{z} \in \mathcal{P}_{d(z)}\left(\Omega_{z}\right)}\left\|f-f_{z}\right\|_{L^{2}\left(\Omega_{z}\right)}^{2}\right)^{1 / 2} .
\end{aligned}
$$

In the infimum, " $\left.v\right|_{\Gamma_{D}}=u_{D}$ " stands for all $v \in H^{1}(\Omega)$ with $v=u_{D}$ on $\Gamma_{D}$.

Proof. Abbreviate $e:=u-u_{h}$ and let $q_{h} \in \mathcal{S}^{k+1}(\mathcal{T})^{d}$. Assume that $v \in H^{1}(\Omega)$ satisfies $v=u_{D}$ on $\Gamma_{D}$ and $\left\|\nabla\left(u_{h}-v\right)\right\|_{2} \leq\|\nabla e\|_{2}$. Then (4.1)-(4.4) imply (3.1). Hence, we may choose $q=q_{h}$ and $w=u-v$ in Theorem [3.1 to obtain (with Cauchy's inequality for the second term in the right-hand side of the subsequent 
equality) that

$$
\begin{aligned}
\|\nabla e\|_{2}^{2}= & \int_{\Omega} \nabla e \cdot \nabla w d x+\int_{\Omega} \nabla e \cdot \nabla\left(v-u_{h}\right) d x \\
\leq & \|\nabla w\|_{2}\left(c_{4}\left\|\nabla u_{h}-q_{h}\right\|_{2}+c_{7}\left\|h_{\mathcal{E}}^{1 / 2}\left(g-q_{h} \cdot n\right)\right\|_{2, \Gamma_{N}}\right. \\
& \left.\quad+c_{5}\left(\sum_{z \in \mathcal{K}} h_{z}^{2} \min _{f_{z} \in \mathcal{P}_{d(z)}\left(\Omega_{z}\right)}\left\|f+\operatorname{div} q_{h}-f_{z}\right\|_{2, \Omega_{z}}^{2}\right)^{1 / 2}\right) \\
& +\left\|\nabla\left(u_{h}-v\right)\right\|_{2}\|\nabla e\|_{2} .
\end{aligned}
$$

Since $\|\nabla w\|_{2} \leq\|\nabla e\|_{2}+\left\|\nabla\left(u_{h}-v\right)\right\|_{2} \leq 2\|\nabla e\|_{2}$, we can divide (4.6) by $\|\nabla e\|_{2}$ to verify

$$
\begin{aligned}
\|\nabla e\|_{2} \leq 2 c_{4} \| \nabla u_{h}- & q_{h}\left\|_{2}+2 c_{7}\right\| h_{\mathcal{E}}^{1 / 2}\left(g-q_{h} \cdot n\right)\left\|_{2, \Gamma_{N}}+\right\| \nabla\left(u_{h}-v\right) \|_{2} \\
& +2 c_{5}\left(\sum_{z \in \mathcal{K}} h_{z}^{2} \min _{f_{z} \in \mathcal{P}_{d(z)}\left(\Omega_{z}\right)}\left\|f+\operatorname{div} q_{h}-f_{z}\right\|_{2, \Omega_{z}}^{2}\right)^{1 / 2} .
\end{aligned}
$$

Let $\operatorname{div} \mathcal{T}$ denote the $\mathcal{T}$-piecewise action of the div-operator. The triangle inequality in the last summand in (4.7) and $h_{z} \lesssim h_{T}$ for $z \in T \cap \mathcal{N}$ and $T \in \mathcal{T}$, a summation over elements, and $\operatorname{div}\left(\mathcal{P}_{d(z)+1}\left(\Omega_{z}\right)^{d}\right) \subseteq \mathcal{P}_{d(z)}\left(\Omega_{z}\right)$ show that

$$
\begin{aligned}
\sum_{z \in \mathcal{K}} h_{z}^{2} & \min _{f_{z} \in \mathcal{P}_{d(z)}\left(\Omega_{z}\right)}\left\|f+\operatorname{div} q_{h}-f_{z}\right\|_{2, \Omega_{z}}^{2} \\
\leq & 3\left\|h_{\mathcal{T}} \operatorname{div} \mathcal{T}\left(\nabla u_{h}-q_{h}\right)\right\|_{2}^{2} \\
& +3 \sum_{z \in \mathcal{K}} h_{z}^{2} \min _{f_{z} \in \mathcal{P}_{d(z)}\left(\Omega_{z}\right)}\left\|f-f_{z}\right\|_{2, \Omega_{z}}^{2} \\
& +3 \sum_{z \in \mathcal{K}} \min _{q_{z} \in \mathcal{P}_{d(z)+1}\left(\Omega_{z}\right)^{d}}\left\|h_{z} \operatorname{div} \mathcal{T}\left(\nabla u_{h}-q_{z}\right)\right\|_{2, \Omega_{z}}^{2} .
\end{aligned}
$$

A $\mathcal{T}$-elementwise inverse estimate shows

$$
\left\|h_{\mathcal{T}} \operatorname{div}_{\mathcal{T}}\left(\nabla u_{h}-q_{h}\right)\right\|_{2} \lesssim\left\|\nabla u_{h}-q_{h}\right\|_{2}
$$

and

$$
\left\|h_{z} \operatorname{div}_{\mathcal{T}}\left(\nabla u_{h}-q_{z}\right)\right\|_{2, \Omega_{z}} \lesssim\left\|\nabla u_{h}-q_{z}\right\|_{2, \Omega_{z}}
$$

(with constants that depend on the polynomial degrees and on the shape of the finite elements but not on their diameters). Utilising this in (4.7)-(4.8), we deduce (4.5).

The following lemmas show that the terms concerning the right-hand-sides $f, g$, and $u_{D}$ in (4.5) are of higher order, provided the given data functions are smooth enough.

Lemma 4.1. For all $z \in \mathcal{K}$, there exists an $h_{z}$-independent constant $c_{13}>0$ such that, if $\left.f\right|_{\Omega_{z}} \in H^{d(z)+1}\left(\Omega_{z}\right)$, we have $\left(D^{d(z)+1} f=\left(\partial^{\alpha} f\right)_{|\alpha|=d(z)+1}\right.$ denotes the vector of all partial derivatives of order $d(z)+1)$

$$
\min _{f_{z} \in \mathcal{P}_{d(z)}\left(\Omega_{z}\right)}\left\|f-f_{z}\right\|_{L^{2}\left(\Omega_{z}\right)} \leq c_{13} h_{z}^{d(z)+1}\left\|D^{d(z)+1} f\right\|_{L^{2}\left(\Omega_{z}\right)} .
$$

Proof. The proof of the lemma can be found, e.g., in BS. 
For a function $w \in H^{1}(\gamma)$ we denote by $\partial w / \partial t$ and $\partial w / \partial s$ its gradient with respect to the arc-length along the $(d-2)$ - respectively $(d-1)$-dimensional submanifold $\gamma$.

Lemma 4.2. Let $E \in \mathcal{E}_{N}$ and assume $\left.g\right|_{E} \in H^{d_{E}}(E)$ for some $d_{E} \geq 1$. Then there exist $q_{h} \in \mathcal{L}^{d_{E}}\left(\omega_{E}\right)^{d}$ and a constant $c_{14}>0$ that is independent of $h_{E}$ and $g$, such that, for $g_{h}:=q_{h} \cdot n$, we have

$$
\left\|g-g_{h}\right\|_{L^{2}(E)} \leq c_{14} h_{E}^{d_{E}}\left\|\partial^{d_{E}} g / \partial s^{d_{E}}\right\|_{L^{2}(E)} .
$$

Proof. The statement is the $(d-1)$-dimensional version of the previous lemma.

Lemma 4.3. Let $u_{D} \in H^{1}\left(\Gamma_{D}\right) \cap C\left(\Gamma_{D}\right)$ satisfy $\left.u_{D}\right|_{E} \in H^{d_{E}+1}(E)$ for some $d_{E} \geq$ $1, E \in \mathcal{E}_{D}$, and $u_{h}(z)=u_{D}(z)$ for all $z \in \mathcal{N} \cap \Gamma_{D}$. If $u_{h}$ also satisfies

$$
\left\|\partial^{\ell}\left(u_{h}-u_{D}\right) / \partial s^{\ell}\right\|_{L^{2}(E)} \leq c_{15} h_{E}^{d_{E}+1-\ell}\left\|\partial^{d_{E}+1} u_{D} / \partial s^{d_{E}+1}\right\|_{L^{2}(E)}
$$

for all $E \in \mathcal{E}_{D}$ and $\ell=0,1,2$ with an $h_{\mathcal{E}}$-independent constant $c_{15}>0$, then there exists an $h_{\mathcal{E}}$-independent constant $c_{16}>0$ such that

$$
\inf _{v \mid \Gamma_{D}=u_{D}}\left\|\nabla\left(v-u_{h}\right)\right\|_{L^{2}(\Omega)} \leq c_{16}\left(\sum_{E \in \mathcal{E}_{D}} h_{E}^{2 d_{E}+1}\left\|\partial^{d_{E}+1} u_{D} / \partial s^{d_{E}+1}\right\|_{L^{2}(E)}^{2}\right)^{1 / 2} .
$$

Proof. The trace theorems yield (both infima are attained)

$$
\inf _{\substack{\left.v \in H^{1}(\Omega) \\ v\right|_{D}=u_{D}}}\left\|\nabla\left(u_{h}-v\right)\right\|_{2}=\inf _{\substack{\left.\tilde{v} \in H^{1}(\Omega) \\ \tilde{v}\right|_{\Gamma_{D}}=\left.u_{h}\right|_{\Gamma_{D}}-u_{D}}}\|\nabla \tilde{v}\|_{2} \lesssim\left\|u_{h}-u_{D}\right\|_{H^{1 / 2}\left(\Gamma_{D}\right)} .
$$

The norm in $H^{1 / 2}\left(\Gamma_{D}\right)$ is equivalent to the norm of minimal extension to $\Gamma$, and so (4.13) leads to

$$
\inf _{\substack{\left.v \in H^{1}(\Omega) \\ v\right|_{\Gamma_{D}}=u_{D}}}\left\|\nabla\left(u_{h}-v\right)\right\|_{2} \lesssim \inf _{\substack{\left.w \in H^{1 / 2}(\Gamma) \\ w\right|_{\Gamma_{D}}=\left.u_{h}\right|_{\Gamma_{D}}-u_{D}}}\|w\|_{H^{1 / 2}(\Gamma)} .
$$

We construct an extension $w$ of $\left.u_{h}\right|_{\Gamma_{D}}-u_{D}$ to $\Gamma$. For $d=2$ we extend $\left.u_{h}\right|_{\Gamma_{D}}-u_{D}$ by zero to $\Gamma$ and obtain a continuous extension $w$. For $d=3$ this extension might be discontinuous, which forces a modification on edges near $\Gamma_{D}$. For $E \in \mathcal{E} \backslash \mathcal{E}_{D}$ with $E \cap \Gamma_{D}=\emptyset$ or $E \cap \Gamma_{D}=\{z\}$ for some $z \in \mathcal{N}$, set $\left.w\right|_{E}=0$. In the remaining cases $E \in \mathcal{E} \backslash \mathcal{E}_{D}$ with $E \cap \Gamma_{D}=\bigcup_{j=1}^{J} E \cap F_{j}=\bigcup_{j=1}^{J} \operatorname{conv}\left\{a_{j}, b_{j}\right\}$ with $J \in\{1,2,3\}$, $F_{j} \in \mathcal{E}_{D}$, and $a_{j}, b_{j} \in \mathcal{N} \cap F_{j}, a_{j} \neq b_{j}, j=1, \ldots, J$, we proceed as follows. Let $s_{E}$ denote the center of inertia of $E$. On each triangle $G_{j}:=\operatorname{conv}\left\{a_{j}, b_{j}, s_{E}\right\}$ we let $w$ be an harmonic extension of $\left.u_{h}\right|_{\Gamma_{D}}-u_{D}$ from $S_{j}:=\operatorname{conv}\left\{a_{j}, b_{j}\right\}$ to $G_{j}$ such that $\left.w\right|_{\partial G_{j} \backslash S_{j}}=0$. Note that $w$ is continuous on $G_{j}$. An interpolation argument and $\|w\|_{2, \partial G_{j}} \leq h_{E}\|\partial w / \partial t\|_{2, \partial G_{j}}$ reveal that

$$
\begin{aligned}
\|\partial w / \partial s\|_{2, G_{j}}^{2} & \lesssim\|w\|_{H^{1 / 2}\left(\partial G_{j}\right)}^{2} \lesssim\|w\|_{2, \partial G_{j}}\|w\|_{H^{1}\left(\partial G_{j}\right)} \leq h_{E}\|\partial w / \partial t\|_{2, \partial G_{j}}^{2} \\
& =h_{E}\left\|\partial\left(u_{h}-u_{D}\right) / \partial t\right\|_{2, S_{j}}^{2} .
\end{aligned}
$$

By a trace theorem [CF], [BS] for the tangential gradient $\partial\left(u_{h}-u_{D}\right) / \partial s$ from $F_{j}$ to $G_{j} \cap F_{j}=S_{j}$ we have

$$
\begin{aligned}
& h_{E}\left\|\partial\left(u_{h}-u_{D}\right) / \partial t\right\|_{2, S_{j}}^{2} \\
& \quad \lesssim\left\|\partial\left(u_{h}-u_{D}\right) / \partial s \cdot t\right\|_{2, F_{j}}^{2}+h_{E}^{2}\left\|\partial\left(\partial\left(u_{h}-u_{D}\right) / \partial s \cdot t\right) / \partial s\right\|_{2, F_{j}}^{2},
\end{aligned}
$$


where $t$ denotes the unit tangent vector along $S_{j}$. Since $t$ is constant, this shows that

$$
h_{E}\left\|\partial\left(u_{h}-u_{D}\right) / \partial t\right\|_{2, S_{j}}^{2} \lesssim\left\|u_{h}-u_{D}\right\|_{H^{1}\left(F_{j}\right)}^{2}+h_{E}^{2}\left\|u_{h}-u_{D}\right\|_{H^{2}\left(F_{j}\right)}^{2} .
$$

Applying (4.15) in (4.14), we find that

$$
\|\partial w / \partial s\|_{2, G_{j}}^{2} \lesssim\left\|u_{h}-u_{D}\right\|_{H^{1}\left(F_{j}\right)}^{2}+h_{E}^{2}\left\|u_{h}-u_{D}\right\|_{H^{2}\left(F_{j}\right)}^{2}
$$

and, using Friedrichs' inequality $\|w\|_{2, G_{j}} \lesssim h_{E}\|\partial w / \partial s\|_{2, G_{j}}$,

$$
\|w\|_{2, G_{j}}^{2} \lesssim h_{E}^{2}\left\|\left(u_{h}-u_{D}\right)\right\|_{H^{1}\left(F_{j}\right)}^{2}+h_{E}^{4}\left\|\left(u_{h}-u_{D}\right)\right\|_{H^{2}\left(F_{j}\right)}^{2} .
$$

Note that the extension is continuous and affects neighbouring edges of $\Gamma_{D}$ only.

Let $w_{1}, \ldots, w_{n}$ denote the nonvanishing functions amongst $\left(\left.w \varphi_{z}\right|_{\Gamma}: z \in \mathcal{N} \cap \Gamma\right)$. By an interpolation estimate and Friedrichs' inequality we infer that, for each $j=$ $1, \ldots, n$,

$$
\begin{aligned}
\left\|w_{j}\right\|_{H^{1 / 2}(\Gamma)}^{2} & \lesssim\left\|w_{j}\right\|_{2, \Gamma}\left\|w_{j}\right\|_{H^{1}(\Gamma)} \\
& \leq\left\|w_{j}\right\|_{2, \Gamma}\left(\left\|w_{j}\right\|_{2, \Gamma}+\left\|\partial w_{j} / \partial s\right\|_{2, \Gamma} \lesssim\left\|h_{\mathcal{E}}^{1 / 2} \partial w_{j} / \partial s\right\|_{2, \Gamma}^{2} .\right.
\end{aligned}
$$

To localise the $H^{1 / 2}$-norm on $\Gamma_{D}$, we employ the arguments from CMS. It was shown therein that there exists a partition $\left(I_{\ell}: \ell=1, \ldots, L\right)$ of $\{1, \ldots, n\}$ such that $w_{j} w_{k}=0$ for any distinct $j, k \in I_{\ell} . L$ is independent of $h_{\mathcal{T}}$, and for each $\ell=1, \ldots, L$ we have

$$
\left\|\sum_{j \in I_{\ell}} w_{j}\right\|_{H^{1 / 2}(\Gamma)}^{2} \lesssim \sum_{j \in I_{\ell}}\left\|w_{j}\right\|_{H^{1 / 2}(\Gamma)}^{2} .
$$

Using the inequality $\left(\sum_{\ell=1}^{L} a_{\ell}\right)^{2} \leq L \sum_{\ell=1}^{L} a_{\ell}^{2}$ and (4.19), we deduce that

$$
\begin{aligned}
\|w\|_{H^{1 / 2}(\Gamma)}^{2} & \leq L \sum_{\ell=1}^{L}\left\|\sum_{j \in I_{\ell}} w_{j}\right\|_{H^{1 / 2}(\Gamma)}^{2} \lesssim L \sum_{\ell=1}^{L} \sum_{j \in I_{\ell}}\left\|w_{j}\right\|_{H^{1 / 2}(\Gamma)}^{2} \\
& \lesssim L \sum_{\ell=1}^{L} \sum_{j \in I_{\ell}}\left\|h_{\mathcal{E}}^{1 / 2} \partial w_{j} / \partial s\right\|_{2, \Gamma}^{2} .
\end{aligned}
$$

With $w_{j}=w \varphi_{z},\left|\partial w_{j} / \partial s\right| \leq|\partial w / \partial s|+|w|\left|\partial \varphi_{z} / \partial s\right| \lesssim|\partial w / \partial s|+|w| / h_{E}$, and $L \lesssim 1$ this shows that

$$
\begin{aligned}
\|w\|_{H^{1 / 2}(\Gamma)} & \lesssim \sum_{\ell=1}^{J} \sum_{j \in I_{\ell}}\left(\left\|h_{\mathcal{E}}^{1 / 2} \partial w / \partial s\right\|_{2, \operatorname{supp} w_{j}}^{2}+\left\|h_{\mathcal{E}}^{-1 / 2} w\right\|_{2, \operatorname{supp} w_{j}}^{2}\right) \\
& \lesssim\left\|h_{\mathcal{E}}^{1 / 2} \partial w / \partial s\right\|_{2, \Gamma}^{2}+\left\|h_{\mathcal{E}}^{-1 / 2} w\right\|_{2, \Gamma}^{2} .
\end{aligned}
$$

Utilising $\left.w\right|_{E}=\left.\left(\left.u_{h}\right|_{\Gamma_{D}}-u_{D}\right)\right|_{E}$ for all $E \in \mathcal{E}_{D}$, and the estimates (4.16) and (4.17), we infer

$$
\begin{aligned}
\|w\|_{H^{1 / 2}(\Gamma)}^{2} \lesssim & \left\|h_{\mathcal{E}}^{-1 / 2}\left(u_{h}-u_{D}\right)\right\|_{2, \Gamma_{D}}^{2}+\left\|h_{\mathcal{E}}^{1 / 2} \partial\left(u_{h}-u_{D}\right) / \partial s\right\|_{2, \Gamma_{D}}^{2} \\
& +\left\|h_{\mathcal{E}}^{3 / 2} \partial_{\mathcal{E}}^{2}\left(u_{h}-u_{D}\right) / \partial s^{2}\right\|_{2, \Gamma_{D}}^{2} .
\end{aligned}
$$

Estimating the summands in the right-hand side of (4.22) on each $E \in \mathcal{E}_{D}$ by (4.11), we conclude the proof. 
Theorem 4.2. Suppose that $\mathcal{S}$ satisfies the hypothesis of Lemma 3.1 and let $d_{E}$, $E \in \mathcal{E}$, be nonnegative integers with $d_{E} \geq 1$ for $E \in \mathcal{E}_{N} \cup \mathcal{E}_{D}$. Assume $\left.f\right|_{\Omega_{z}} \in$ $H^{d(z)+1}\left(\Omega_{z}\right)$ for all $z \in \mathcal{K},\left.g\right|_{E} \in H^{d_{E}}(E)$ for all $E \in \mathcal{E}_{N}$, and $\left.u_{D}\right|_{E} \in H^{d_{E}+1}(E)$ for all $E \in \mathcal{E}_{D}$. Then, if $u_{h}$ satisfies the hypothesis of Lemma 4.3, there exists $g_{h} \in L^{\infty}\left(\Gamma_{N}\right)$ with $\left.g_{h}\right|_{E} \in \mathcal{P}_{d_{E}}(E)$ for all $E \in \mathcal{E}_{N}$, such that

$$
\begin{aligned}
\left\|\nabla\left(u-u_{h}\right)\right\|_{L^{2}(\Omega)} \leq & c_{17}\left(\sum_{E \in \mathcal{E}_{\Omega} \cup \mathcal{E}_{N}} \min _{\begin{array}{c}
q_{E} \in \mathcal{P}_{d_{E}}\left(\omega_{E}\right)^{d}, \\
q_{E} \cdot n=g_{h} \text { on } E \cap \Gamma_{N}
\end{array}}\left\|\nabla u_{h}-q_{E}\right\|_{L^{2}\left(\omega_{E}\right)}^{2}\right)^{1 / 2} \\
& +2 c_{7} c_{14}\left(\sum_{E \in \mathcal{E}_{N}} h_{E}^{2 d_{E}+1}\left\|\partial^{d_{E}} g / \partial s^{d_{E}}\right\|_{L^{2}(E)}^{2}\right)^{1 / 2} \\
& +c_{16}\left(\sum_{E \in \mathcal{E}_{D}} h_{E}^{2 d_{E}+1}\left\|\partial^{d_{E}+1} u_{D} / \partial s^{d_{E}+1}\right\|_{L^{2}(E)}^{2}\right)^{1 / 2} \\
& +2 c_{5} c_{13}\left(\sum_{z \in \mathcal{K}} h_{z}^{2(d(z)+2)}\left\|D^{d(z)+1} f\right\|_{L^{2}\left(\Omega_{z}\right)}^{2}\right)^{1 / 2}
\end{aligned}
$$

The constant $c_{17}>0$ depends only on the shapes of the elements and the polynomial degrees $d_{E}$ and $d_{z}$.

Proof. Let $g_{h} \in L^{\infty}\left(\Gamma_{N}\right)$ be such that, for all $E \in \mathcal{E}_{N},\left.g_{h}\right|_{E} \in \mathcal{P}_{d_{E}}(E)$ is an approximation of $\left.g\right|_{E}$ as in Lemma 4.2 Choose $k \in \mathbb{N}_{0}$ such that $\nabla u_{h} \in \mathcal{L}^{k}(\mathcal{T})$ and $g_{h} \in \mathcal{L}^{k+1}\left(\mathcal{E}_{N}\right)$. Utilising Theorem 4.1 we verify

$$
\begin{aligned}
\left\|\nabla\left(u-u_{h}\right)\right\|_{2} & \\
\leq & \min _{q_{h} \in \mathcal{S}^{k+1}(\mathcal{T})^{d}}\left(c_{11}\left\|\nabla u_{h}-q_{h}\right\|_{2}+2 c_{7}\left\|h_{\mathcal{E}}^{1 / 2}\left(g_{h}-q_{h} \cdot n\right)\right\|_{2, \Gamma_{N}}\right) \\
& +\left\|h_{\mathcal{E}}^{1 / 2}\left(g-g_{h}\right)\right\|_{2, \Gamma_{N}}+c_{12}\left(\sum_{z \in \mathcal{K}} \min _{q_{z} \in \mathcal{P}_{d(z)+1}\left(\Omega_{z}\right)^{d}}\left\|\nabla u_{h}-q_{z}\right\|_{2, \Omega_{z}}^{2}\right)^{1 / 2} \\
& +\inf _{\left.v\right|_{\Gamma_{D}}=u_{D}}\left\|\nabla\left(u_{h}-v\right)\right\|_{2}+2 c_{5}\left(\sum_{z \in \mathcal{K}} h_{z}^{2} \min _{f_{z} \in \mathcal{P}_{d(z)}\left(\Omega_{z}\right)}\left\|f-f_{z}\right\|_{2, \Omega_{z}}^{2}\right)^{1 / 2} .
\end{aligned}
$$

By Lemma 3.1 and since each inner edge belongs to a finite number of patches, we have

$$
\begin{aligned}
& \sum_{z \in \mathcal{K}} \min _{q_{z} \in \mathcal{P}_{d(z)+1}\left(\Omega_{z}\right)^{d}}\left\|\nabla u_{h}-q_{z}\right\|_{2, \Omega_{z}}^{2} \\
& \quad \leq c_{9} \sum_{z \in \mathcal{K}} \sum_{E \in \mathcal{E}_{\Omega_{z}}} \min _{q_{E} \in \mathcal{P}_{d_{E}}\left(\omega_{E}\right)^{d}}\left\|\nabla u_{h}-q_{E}\right\|_{2, \omega_{E}}^{2} \\
& \quad \lesssim \sum_{E \in \mathcal{E}_{\Omega}} \min _{q_{E} \in \mathcal{P}_{d_{E}}\left(\omega_{E}\right)^{d}}\left\|\nabla u_{h}-q_{E}\right\|_{2, \omega_{E}}^{2} .
\end{aligned}
$$


With Lemma 3.2 and noting that for all $E \in \mathcal{E}_{N}$ there exists $q_{E} \in \mathcal{P}_{d_{E}}\left(\omega_{E}\right)^{d}=$ $\mathcal{L}^{d_{E}}\left(\omega_{E}\right)^{d}$ with $\left.q_{E}\right|_{E} \cdot n=\left.g_{h}\right|_{E}$, we can estimate

$$
\begin{aligned}
& \min _{q_{h} \in \mathcal{S}^{k+1}(\mathcal{T})^{d}}\left(\left\|\nabla u_{h}-q_{h}\right\|_{2}^{2}+\left\|h_{\mathcal{E}}^{1 / 2}\left(g_{h}-q_{h} \cdot n\right)\right\|_{2, \Gamma_{N}}^{2}\right) \\
& \quad \leq c_{10} \sum_{E \in \mathcal{E}_{\Omega} \cup \mathcal{E}_{N}} \min _{q_{E} \in \mathcal{P}_{d_{E}}\left(\omega_{E}\right)^{d}}\left(\left\|\nabla u_{h}-q_{E}\right\|_{2, \omega_{E}}^{2}+h_{E}\left\|g_{h}-q_{E} \cdot n\right\|_{2, E \cap \Gamma_{N}}^{2}\right) \\
& \quad \leq c_{10} \sum_{E \in \mathcal{E}_{\Omega} \cup \mathcal{E}_{N}} \min _{\substack{q_{E} \in \mathcal{P}_{d_{E}}\left(\omega_{E}\right)^{d}, q_{E} \cdot n=g_{h} \text { on } E \cap \Gamma_{N}}}\left\|\nabla u_{h}-q_{E}\right\|_{2, \omega_{E}}^{2} .
\end{aligned}
$$

Estimating the first and the third term in (4.24) by (4.25) resp. (4.26) and utilising Lemmas 4.14 .3 to bound the remaining terms in (4.24) yields (4.23).

The a posteriori error estimate given in the previous theorem is efficient up to higher order terms which depend on the smoothness of $\nabla u$, as the following theorem shows.

Theorem 4.3. Let $g_{h} \in \mathcal{L}^{k}\left(\mathcal{E}_{N}\right)$ with $\left.g_{h}\right|_{E} \in \mathcal{P}_{d_{E}}(E)$ for $d_{E} \in\{0,1, \ldots, k\}$ on each $E \in \mathcal{E}_{N}$. Then we have

$$
\begin{aligned}
& \left(\sum_{E \in \mathcal{E}_{\Omega} \cup \mathcal{E}_{N}} \min _{\substack{q_{E} \in \mathcal{P}_{d_{E}}\left(\omega_{E}\right)^{d}, q_{E} \cdot n=g_{h} \text { on } E \cap \Gamma_{N}}}\left\|\nabla u_{h}-q_{E}\right\|_{L^{2}\left(\omega_{E}\right)}^{2}\right)^{1 / 2} \\
& \leq \sqrt{2 d}\left\|\nabla\left(u-u_{h}\right)\right\|_{L^{2}(\Omega)} \\
& \quad+\left(\sum_{E \in \mathcal{E}_{\Omega} \cup \mathcal{E}_{N}} \min _{\substack{q_{E} \in \mathcal{P}_{d_{E}}\left(\omega_{E}\right)^{d}, q_{E} \cdot n=g_{h} \text { on } E \cap \Gamma_{N}}}\left\|\nabla u-q_{E}\right\|_{L^{2}\left(\omega_{E}\right)}^{2}\right)^{1 / 2} .
\end{aligned}
$$

Proof. Since $g_{h} \in \mathcal{P}_{d_{E}}(E)$ for each $E \in \mathcal{E}_{N}$, the minima in the left-hand side of (4.27) are well defined. We infer, with Young's inequality and an arbitrary $\gamma>0$,

$$
\begin{aligned}
& \sum_{E \in \mathcal{E}_{\Omega} \cup \mathcal{E}_{N}} \min _{\substack{q_{E} \in \mathcal{P}_{d_{E}}\left(\omega_{E}\right)^{d}, q_{E} \cdot n=g_{h} \text { on } \\
E \cap \Gamma_{N}}}\left\|\nabla u_{h}-q_{E}\right\|_{2, \omega_{E}}^{2} \\
& \leq \sum_{E \in \mathcal{E}_{\Omega} \cup \mathcal{E}_{N}} \min _{\substack{q_{E} \in \mathcal{P}_{d_{E}}\left(\omega_{E}\right)^{d}, q_{E} \cdot n=g_{h} \text { on } \\
E \cap \Gamma_{N}}}\left(\left\|\nabla\left(u_{h}-u\right)\right\|_{2, \omega_{E}}+\left\|\nabla u-q_{E}\right\|_{2, \omega_{E}}\right)^{2} \\
& \leq(1+1 / \gamma) \sum_{E \in \mathcal{E}_{\Omega} \cup \mathcal{E}_{N}}\left\|\nabla\left(u-u_{h}\right)\right\|_{2, \omega_{E}}^{2} \\
& \quad+(1+\gamma) \sum_{E \in \mathcal{E}_{\Omega} \cup \mathcal{E}_{N}} \min _{\substack{q_{E} \in \mathcal{P}_{d_{E}}\left(\omega_{E}\right)^{d}, q_{E} \cdot n=g_{h} \text { on } E \cap \Gamma_{N}}}\left\|\nabla u-q_{E}\right\|_{2, \omega_{E}}^{2} .
\end{aligned}
$$

Rearranging the sum over the edges $E \in \mathcal{E}_{\Omega} \cup \mathcal{E}_{N}$ and using the fact that $T \in \mathcal{T}$ has at most $2 d$ edges, the first term in the right-hand side of (4.28) can be estimated by

$$
\sum_{T \in \mathcal{T}} \sum_{\substack{E \subset \partial \mathcal{E}_{D} \\ E \notin \mathcal{E}_{D}}}\left\|\nabla\left(u-u_{h}\right)\right\|_{2, T}^{2} \leq 2 d\left\|\nabla\left(u-u_{h}\right)\right\|_{2, \Omega}^{2} .
$$


A direct calculation shows that $(1+1 / \gamma) a^{2}+(1+\gamma) b^{2}$, where $a, b>0$, is minimised for $\gamma=b / a$ with minimal value $a^{2}+2 a b+b^{2}=(a+b)^{2}$. The optimal choice for $\gamma$ in the right-hand side of (4.28) yields (4.27).

Remark 4.1. The local degrees $d_{E}$ have to be chosen large enough to obtain higher order terms in the reliability estimate (4.23) and in the efficiency estimate (4.27) at the same time.

Remark 4.2. The definition of the approximation operator $\mathcal{J}$ is related to "partition of unity finite elements" $\mathrm{MBa}$, where for a fixed nonnegative integer $\ell$,

$$
\mathcal{S}=\left\{\sum_{z \in \mathcal{N}} q_{z} \varphi_{z}: q_{z} \in \mathcal{P}_{\ell}\left(\omega_{z}\right)\right\}
$$

(so that $d(z)=\ell$ for all $z \in \mathcal{K}$ by definition). When $\mathcal{S}$ is given by (4.29), a reasonable choice for $d_{E}$ is $d_{E}=\ell+1, E \in \mathcal{E}$, so that inequality (4.23) reads

$$
\begin{aligned}
\left\|\nabla\left(u-u_{h}\right)\right\|_{L^{2}(\Omega)} \leq & c_{17}\left(\sum_{E \in \mathcal{E}_{\Omega} \cup \mathcal{E}_{N}} \min _{\begin{array}{c}
q_{E} \in \mathcal{P}_{\ell+1}\left(\omega_{E}\right)^{d}, \\
q_{E} \cdot n=g_{h} \text { on } E \cap \Gamma_{N}
\end{array}}\left\|\nabla u_{h}-q_{E}\right\|_{L^{2}\left(\omega_{E}\right)}^{2}\right)^{1 / 2} \\
& +c_{18}\left(\left\|h_{\mathcal{E}}^{\ell+3 / 2} \partial_{\mathcal{E}}^{\ell+2} u_{D} / \partial s^{\ell+2}\right\|_{L^{2}\left(\Gamma_{D}\right)}\right. \\
& \left.\quad+\left\|h_{\mathcal{E}}^{\ell+3 / 2} \partial_{\mathcal{E}}^{\ell+1} g / \partial s^{\ell+1}\right\|_{L^{2}\left(\Gamma_{N}\right)}+\left\|h_{\mathcal{T}}^{\ell+2} D^{\ell+1} f\right\|_{L^{2}(\Omega)}\right)
\end{aligned}
$$

with an $\left(h_{\mathcal{T}}, h_{\mathcal{E}}\right)$-independent constant $c_{18}>0$. Here, $\partial_{\mathcal{E}} \cdot / \partial s$ denotes the edgewise differentiation along $\Gamma_{D}$ and $\Gamma_{N}$.

Remark 4.3. An a posteriori error estimate based on a global averaging technique could be formulated as follows: There exists an $\left(h_{\mathcal{T}}, h_{\mathcal{E}}\right)$-independent constant $c_{19}>$ 0 such that, if $\nabla u_{h} \in \mathcal{L}^{k}(\mathcal{T})^{d}$, we have

$$
\begin{aligned}
\left\|\nabla\left(u-u_{h}\right)\right\|_{L^{2}(\Omega)} & \leq c_{19} \min _{q_{h} \in \mathcal{P}_{m}(\Omega)^{d}}\left(\left\|\nabla u_{h}-q_{h}\right\|_{L^{2}(\Omega)}+\left\|h_{\mathcal{E}}^{1 / 2}\left(q_{h} \cdot n-g\right)\right\|_{L^{2}\left(\Gamma_{N}\right)}\right) \\
& +\inf _{\left.v\right|_{\Gamma_{D}}=u_{D}}\left\|\nabla\left(v-u_{h}\right)\right\|_{L^{2}(\Omega)} \\
& +2 c_{5}\left(\sum_{z \in \mathcal{K}} h_{z}^{2} \min _{f_{z} \in \mathcal{P}_{d(z)}\left(\Omega_{z}\right)}\left\|f-f_{z}\right\|_{L^{2}\left(\Omega_{z}\right)}^{2}\right)^{1 / 2}
\end{aligned}
$$

with $m:=\min \{k\} \cup\{d(z): z \in \mathcal{K}\}+1$. The authors failed to replace $\mathcal{P}_{m}(\Omega)^{d}$ in the minimisation over $q_{h}$ in (4.31) by a larger discrete space (such as certain piecewise polynomials) to make it efficient.

Proof of 4.31. By Theorem 4.1 we only need to estimate the term

$$
\sum_{z \in \mathcal{K}} \min _{q_{z} \in \mathcal{P}_{d(z)+1}\left(\Omega_{z}\right)^{d}}\left\|\nabla u_{h}-q_{z}\right\|_{2, \Omega_{z}}^{2}
$$


by the minimum in (4.31). For this, let $\tilde{q}_{h} \in \mathcal{P}_{m}(\Omega)^{d}$ denote the minimiser in (4.31). Since $\tilde{q}_{z}:=\left.\tilde{q}_{h}\right|_{\Omega_{z}} \in \mathcal{P}_{d(z)+1}\left(\Omega_{z}\right)^{d}$, we can conclude that

$\sum_{z \in \mathcal{K}} \min _{q_{z} \in \mathcal{P}_{d(z)+1}\left(\Omega_{z}\right)^{d}}\left\|\nabla u_{h}-q_{z}\right\|_{2, \Omega_{z}}^{2} \leq \sum_{z \in \mathcal{K}}\left\|\nabla u_{h}-\tilde{q}_{z}\right\|_{2, \Omega_{z}}^{2} \leq c_{19}\left\|\nabla u_{h}-\tilde{q}_{h}\right\|_{2, \Omega}^{2}$.

Remark 4.4. For the restriction $\mathcal{T}_{E}=\left\{T \in \mathcal{T}: T \subseteq \bar{\omega}_{E}\right\}$ of the triangulation $\mathcal{T}$ to $\omega_{E}$, the equivalence

$$
\min _{q_{h} \in \mathcal{S}^{k}(\mathcal{T})^{d}}\left\|p_{h}-q_{h}\right\|_{L^{2}(\Omega)}^{2} \approx \sum_{E \in \mathcal{E}_{\Omega}} \min _{q_{E} \in \mathcal{S}^{k}\left(\mathcal{T}_{E}\right)^{d}}\left\|p_{h}-q_{E}\right\|_{L^{2}\left(\omega_{E}\right)}^{2}
$$

holds for $p_{h} \in \mathcal{L}^{k}(\mathcal{T})^{d}$. For $p_{h} \in \mathcal{L}^{0}(\mathcal{T})^{d}$ we also have (by a compactness argument)

$$
\min _{q_{E} \in \mathcal{S}^{k}\left(\mathcal{T}_{E}\right)^{d}}\left\|p_{h}-q_{E}\right\|_{L^{2}\left(\omega_{E}\right)}^{2} \approx \min _{q_{E} \in \mathcal{P}_{k}\left(\omega_{E}\right)^{d}}\left\|p_{h}-q_{E}\right\|_{L^{2}\left(\omega_{E}\right)}^{2} .
$$

The left-hand side of (4.33) thus defines an efficient (provided $k \geq 1$ ) and reliable error estimate for lowest order finite element methods and $p_{h}=\nabla u_{h}$. However, there is no analogon for higher order finite element methods, since (4.34) is not valid if $p_{h}$ is not elementwise constant.

\section{Numerical EXPERIMENTS}

The theoretical results of this paper are supported by numerical experiments for $d=2$. Here, we report on three examples of the problem (4.1)-(4.3) on uniform, $h-, p$-, $h p$-adapted, and perturbed meshes. Two of these examples were considered in [CB] for lowest order schemes.

Example 5.1. Let $f(x, y):=-8 \pi^{2} \sin (2 \pi x) \sin (2 \pi y)$ on the unit square $\Omega:=$ $(0,1)^{2}$ and set $u_{D}:=0$ on $\Gamma_{D}:=\partial \Omega$. The exact solution is then given by $u(x, y)=\sin (2 \pi x) \sin (2 \pi y)$. In this example the right-hand sides as well as the exact solution are smooth. The coarsest triangulation $\mathcal{T}_{0}$ consists of one square halved by the diagonal parallel to $(1,1)$. The initial polynomial degrees $p^{0}=\left(p_{T}^{0}\right)_{T \in \mathcal{T}_{0}}$ are chosen to be equal to $p$ for all $T \in \mathcal{T}_{0}$ and various values $p=1,2, \ldots, 6$.

Example 5.2. Let $f:=-\Delta u$ for the function

$$
u(x, y):=x(1-x) y(1-y) \arctan (60(r-1))
$$

$r^{2}:=(x-1.25)^{2}+(y+0.25)^{2}$ on the unit square $\Omega:=(0,1)^{2}$, and set $u_{D}:=0$ on the entire boundary $\Gamma_{D}:=\partial \Omega$. The solution $u$ to (4.1)-(4.3) is $H^{2}$-regular but $f$ (although theoretically smooth) has huge gradients on the circle with radius 1 around $(1.25,-0.25)$. The coarsest triangulation $\mathcal{T}_{0}$ consists of four squares halved by diagonals parallel to $(1,1)$. The initial polynomial degrees $p^{0}=\left(p_{T}^{0}\right)_{T \in \mathcal{T}_{0}}$ are chosen to be equal to $p$ for all $T \in \mathcal{T}_{0}$ and various values $p=1,2, \ldots, 6$.

Example 5.3. Let $f:=0$ on the $\mathrm{L}$-shaped domain $\Omega:=(-1,1)^{2} \backslash[0,1] \times[-1,0]$, $u_{D}:=0$ on the Dirichlet boundary $\Gamma_{D}:=\{0\} \times[-1,0] \cup[0,1] \times\{0\}$, and, on the Neumann boundary $\Gamma_{N}:=\partial \Omega \backslash \Gamma_{D}$,

$$
g(r, \varphi):=2 / 3 r^{-1 / 3}(-\sin (\varphi / 3), \cos (\varphi / 3)) \cdot n
$$

using polar coordinates $(r, \varphi)$. The exact solution $u(r, \varphi):=r^{2 / 3} \sin (2 \varphi / 3)$ of (4.1)(4.3) has a typical corner singularity at the origin. In this example, the right-hand sides are smooth, but the solution is not. The coarsest triangulation $\mathcal{T}_{0}$ consists of 


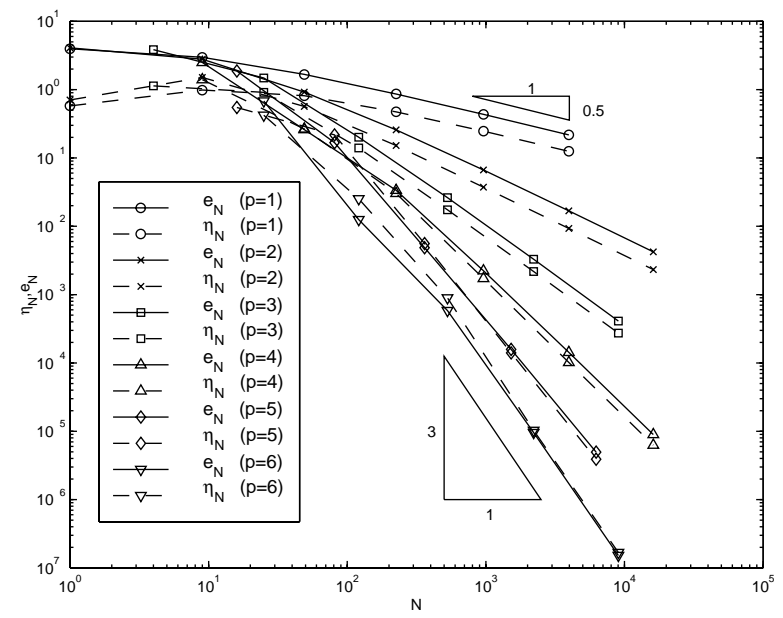

FiguRE 1. Error and error estimator for uniform mesh-refinement in Example 5.1 for different fixed polynomial degrees.

three squares halved by diagonals parallel to $(1,1)$. The initial polynomial degrees $p^{0}=\left(p_{T}^{0}\right)_{T \in \mathcal{T}_{0}}$ are chosen to be equal to $p$ for all $T \in \mathcal{T}_{0}$ and various values $p=1,2, \ldots, 6$.

The following adaptive algorithm generates all the sequences of meshes $\mathcal{T}_{0}, \mathcal{T}_{1}$, $\mathcal{T}_{2}, \ldots$ in this paper which are $h$-uniform for $\Theta=0$ or $h$-adapted for $\Theta=1 / 2$ in (5.2). No element is refined if $\Theta=2$. We also allow raising polynomial degrees steered by the parameter $\delta \in\{(0,0),(1,1),(0,1)\}$. If $\delta=(0,0)$, the initial polynomial degrees are kept on all triangles and their subtriangles. For $\delta=(1,1)$, we increase the degree on each element by one during each iteration of the algorithm while for $\delta=(0,1)$ the polynomial degree is increased only on those triangles that are not $h$-refined during the respective iteration of the algorithm.

Since the resulting meshes might show local symmetries, we considered meshes that are either unperturbed (relative to $\mathcal{T}_{0}$ ) for $\vartheta=0$ or randomly perturbed for $\vartheta=1$ in step (e).

The implementation was performed in Matlab in the spirit of [ACF] with a direct solution of linear systems of equations. For details on the red-blue-greenrefinements we refer to $[\mathrm{V}]$.

Algorithm $\left(A_{\Theta}^{\vartheta, \delta}\right)$. (a) Start with a coarse mesh $\mathcal{T}_{0}$ and polynomial degrees $p^{0}=$ $\left(p_{T}^{0}\right)_{T \in \mathcal{T}_{0}}, k=0$.

(b) Compute the discrete solution $u_{h}$ on the actual mesh $\mathcal{T}_{k}$ in the space

$$
\mathcal{S}^{p^{k}}\left(\mathcal{T}_{k}\right):=\left\{v_{h} \in C(\bar{\Omega}): \forall T \in \mathcal{T},\left.v_{h}\right|_{T} \in P_{T}^{p_{T}^{k}}\right\} .
$$

(c) Set $d_{E}:=\max \left(p_{T_{1}}^{k}, p_{T_{2}}^{k}\right)$ if $E=T_{1} \cap T_{2} \in \mathcal{E}_{\Omega}$ and $d_{E}:=p_{T}^{k}$ if $E=T \cap \Gamma_{N} \in \mathcal{E}_{N}$. Compute, for all $E \in \mathcal{E}_{\Omega} \cup \mathcal{E}_{N}$, error indicators

$$
\eta_{Z, E}:=\frac{1}{\sqrt{3}} \min _{\substack{q_{E} \in \mathcal{P}_{d_{E}}\left(\omega_{E}\right)^{d}, q_{E} \cdot n=g_{h} \text { on } E \cap \Gamma_{N}}}\left\|\nabla u_{h}-q_{E}\right\|_{L^{2}\left(\omega_{E}\right)}
$$



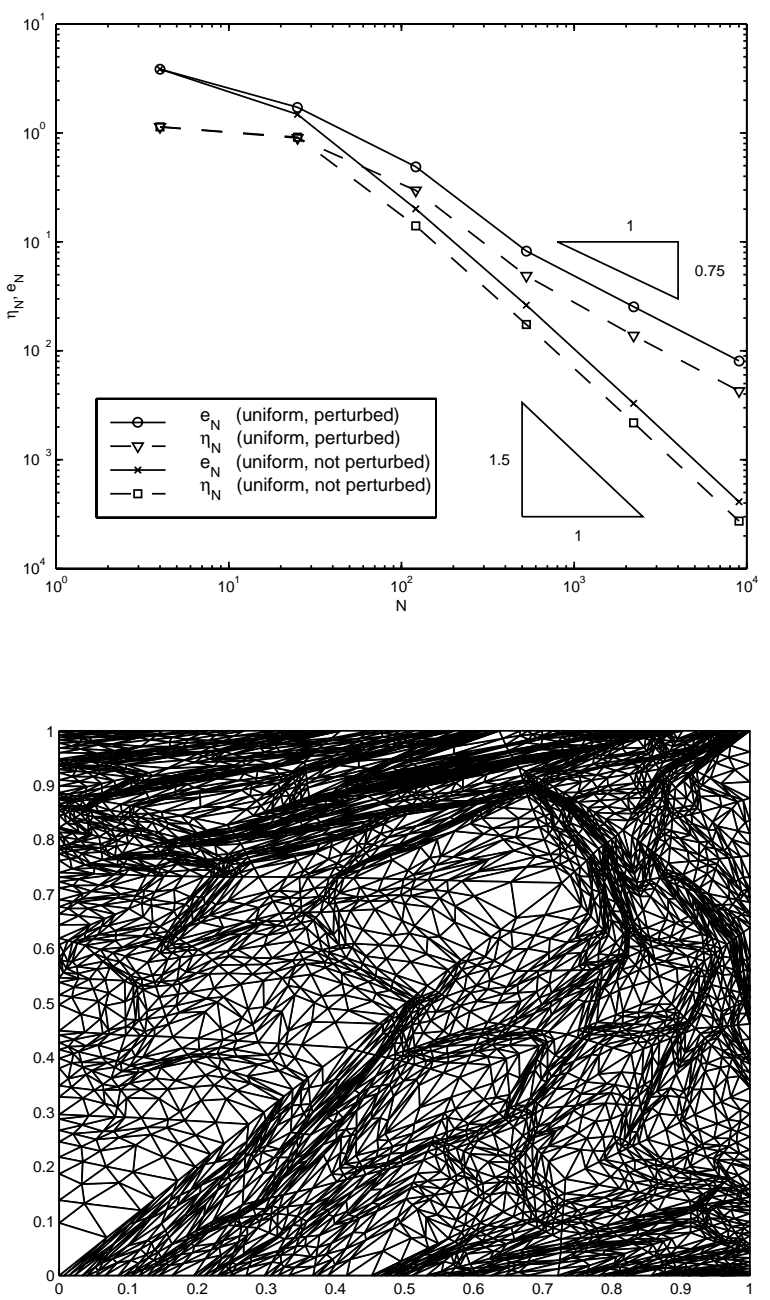

FiguRE 2. Error and error estimator on perturbed meshes for uniform $h$-refinement and fixed polynomial degrees $p=3$ in Example 5.1 (top). Perturbed mesh $\mathcal{I}_{6}$ with 4225 nodes (bottom).

and plot energy error $e_{N}:=\left\|\nabla\left(u-u_{h}\right)\right\|_{L^{2}(\Omega)}$ and its estimator

$$
\eta_{N}^{2}:=\sum_{E \in \mathcal{E}_{\Omega} \cup \mathcal{E}_{N}} \eta_{Z, E}^{2}
$$

versus the degree of freedom $N$ of the triangulation $\mathcal{T}_{k}$.

(d) Mark the edge $E$ for red-refinement provided

$$
\eta_{Z, E} \geq \Theta \max _{E^{\prime} \in \mathcal{E}_{\Omega} \cup \mathcal{E}_{N}} \eta_{Z, E^{\prime}} .
$$

(e) Mark further edges (red-blue-green-refinement) to avoid hanging nodes. Generate a new triangulation $\tilde{\mathcal{T}}_{k+1}$ using edge-midpoints if $\vartheta=0$ and points on the edges at a random distance at most $0.1 h_{E}$ from the edge-midpoints if $\vartheta=1$. For $T \in \tilde{\mathcal{T}}_{k+1}$ set $p_{T}^{k+1}:=p_{T^{\prime}}^{k}+\delta_{1}$ if $T \subsetneq T^{\prime}$ for $T^{\prime} \in \mathcal{T}^{k}$ and define $p_{T}^{k+1}:=p_{T}^{k}+\delta_{2}$ 


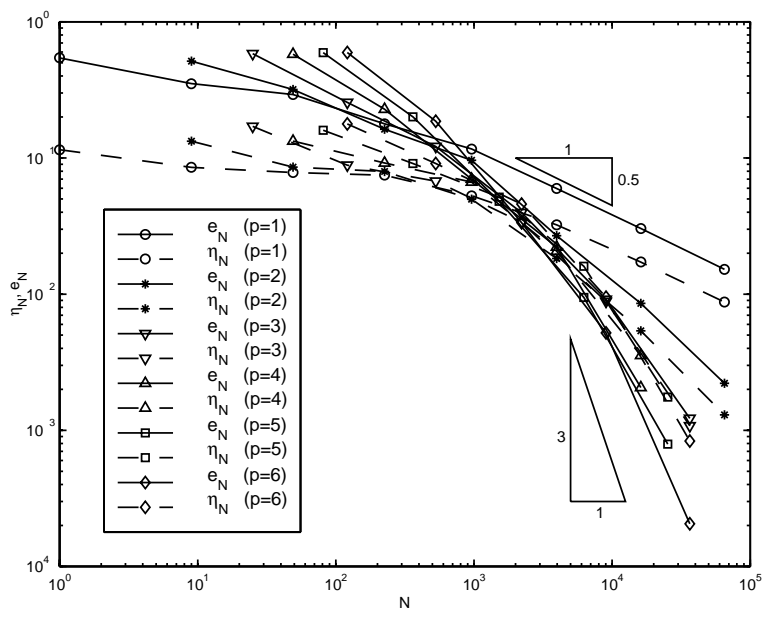

FiguRE 3. Error and error estimator for uniform mesh-refinement in Example 5.2 for different fixed polynomial degrees.

if $T \in \mathcal{T}_{k} \cap \tilde{\mathcal{T}}_{k+1}$. Perturb the nodes $z \in \mathcal{N}_{k+1}$ of the mesh $\tilde{\mathcal{T}}_{k+1}$ at random with values taken uniformly from a ball around $z$ of radius $\vartheta 2^{-k} / 15$. Correct boundary nodes by orthogonal projection onto that boundary piece they are expected such that $\Omega, \Gamma_{D}, \Gamma_{N}$ are matched by the resulting mesh $\mathcal{T}_{k+1}$ exactly. Update $k$ and go to $(\mathrm{b})$.

Remark 5.1. The proof of Theorem 4.3 shows that we may choose the factor $1 / \sqrt{3}$ in (5.1) to obtain an efficient error estimate with constant 1 for our two-dimensional examples with triangles.

Remark 5.2. In our numerical experiments the minimiser in (5.1) is calculated explicitly. The resulting linear system of equations is badly scaled for large polynomial degrees $p$ (we employed transformed Legendre polynomials in such cases). The constraint $q_{E} \cdot n=g_{h}$ is satisfied by matching $q_{E} \cdot n$ and $g$ in nodes on $E$.

We used Algorithm $\left(A_{0}^{0,(0,0)}\right)$ in Example 5.1 with uniform initial polynomial degrees $p_{T}=p$ for all $T \in \mathcal{T}_{0}$, where $p=1,2, \ldots, 6$. Experimental convergence rates are indicted in Figure 1 where we plotted the entries $\left(N, e_{N}\right)$ and $\left(N, \eta_{N}\right)$. A logscaling on both axes allows a slope $-\alpha$ of a straight line that connects subsequent entries to be interpreted as an experimental convergence rate $2 \alpha$ (owing to $N \propto$ $h^{-2}$ in two dimensions). Moreover, we see from Figure 1 that the error estimator $\eta_{N}$ serves as a good approximation for the error $e_{N}$ in this example. When a perturbation of the meshes is introduced we obtain with Algorithms $\left(A_{0}^{0,(0,0)}\right)$ and $\left(A_{0}^{1,(0,0)}\right)$ for polynomial degrees equal to three the results displayed in the top plot of Figure 2 The results become worse for the perturbed meshes but still $\eta_{N}$ approximates $e_{N}$ reasonably. The perturbed mesh $\mathcal{T}_{6}$ with $N=9025$ is shown in the bottom plot of Figure 2 and appears quite degenerate.

The lack of smoothness of the exact solution in Example 5.2 results in a poorer quality of the numerical solution and the estimates than in the previous numerical experiment. Figure 3 shows the results for uniform mesh-refinement and fixed polynomial degrees obtained from Algorithm $\left(A_{0}^{0,(0,0)}\right)$. For polynomial degrees 

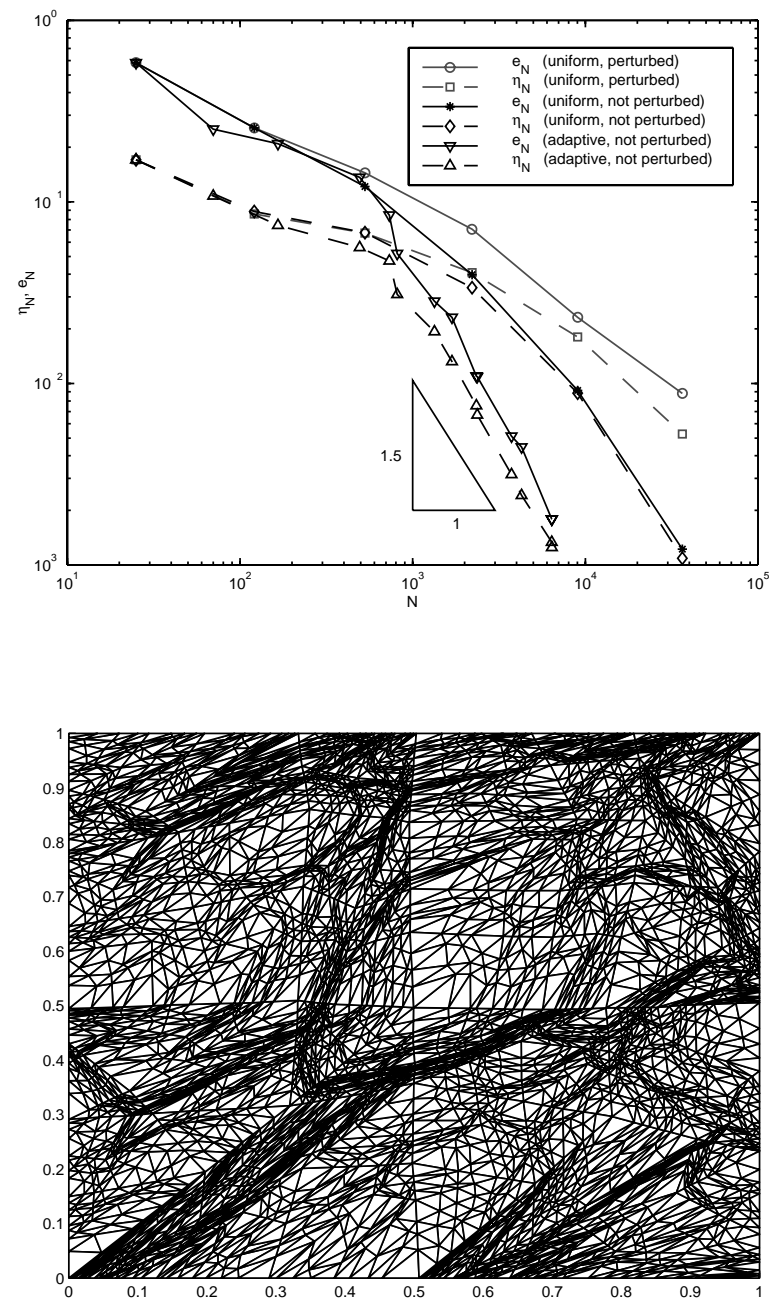

FiguRe 4. Error and error estimator on perturbed meshes for uniform $h$-refinement and fixed polynomial degree $p=3$ in Example 5.2 compared to uniform and $h$-adaptive mesh-refinement without perturbation (top). Perturbed mesh with 4225 nodes (bottom).

$p=1,2,3$ we obtain the expected convergence rates, though the preasymptotic range is very large, while for $p=4,5,6$ the values $\left(N, e_{N}\right)$ and $\left(N, \eta_{N}\right)$ do not result in a straight line in the plot, but the quotient $\eta_{N} / e_{N}$ remains in a small intervall containing one. This is also true for the perturbed meshes in the top and bottom plots of Figure 4, where we chose $p=3$ and used Algorithm $\left(A_{0}^{1,(0,0)}\right)$ to generate the triangulations. The top plot of Figure 4 also shows that an adaptive mesh refinement, realised by Algorithm $\left(A_{1 / 2}^{0,(0,0)}\right)$, yields smaller errors than the uniform mesh-refinement from Algorithm $\left(A_{0}^{0,(0,0)}\right)$ at comparable numbers of degrees of freedom. 


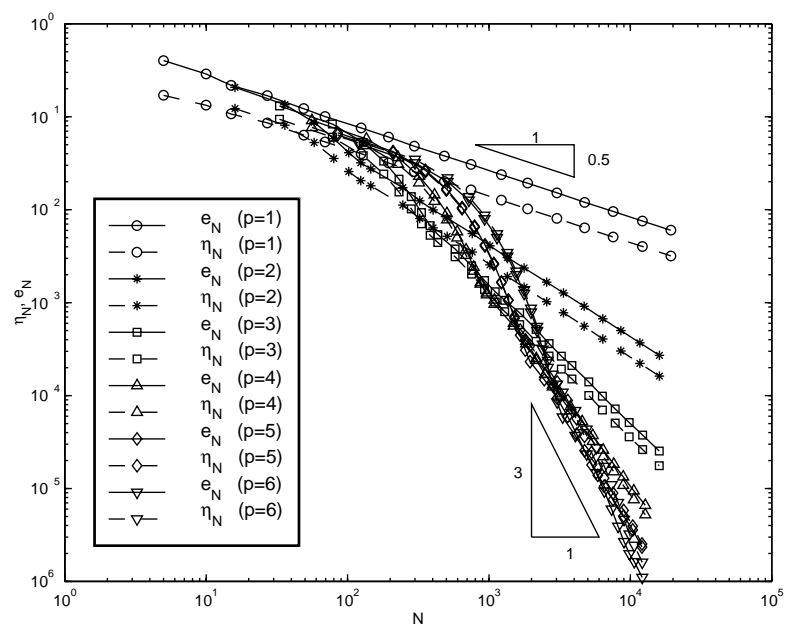

FiguRE 5. Error and error estimator for adaptive meshrefinement in Example 5.3 for different fixed polynomial degrees.

The numerical results for (unperturbed) $h$-adaptive mesh-refinement, i.e., the results obtained with Algorithm $\left(A_{1 / 2}^{0,(0,0)}\right)$, in Example 5.3 for fixed polynomial degrees $p=1,2, \ldots, 6$, are displayed in Figure 5] We obtain optimal experimental convergence rates for $p=1,2, \ldots, 6$. Algorithm $\left(A_{2}^{0,(1,1)}\right)$ ran a $p$-version on a fixed mesh $\mathcal{T}$ with 96 elements obtained by two uniform red-refinements of $\mathcal{T}_{0}$. The top plot of Figure 6 displays the results for the $p$-refinements and $p=1,2, \ldots, 10$. For small $p$ we observe efficiency (i.e., $\eta_{N} \leq e_{N}$ ) which decreases for larger $p$ (e.g., $e_{N}<\eta_{N}$ for $p \geq 6$ ). This is in agreement with our theoretical predictions, as efficiency is linked to the smoothness of the exact solution, which is limited in this example, while the given data $f, g$, and $u_{D}$ are smooth.

For comparison we considered an $h p$-like refinement strategy by running Algorithm $\left(A_{1 / 2}^{0,(0,1)}\right)$. The obtained mesh-refinement and the degree distribution (via different shadings) is given in Figure 5. We observe an automatic geometric refinement towards the origin (where $u$ is singular) and a reverse distribution of the polynomial degrees. This appears as a good strategy: Large elements have a high polynomial degree where the exact solution $u$ is smooth, and small elements of lower degree appear near the singular points of $u$. The corresponding estimate $\eta_{N}$ for $e_{N}$ is satisfactory and even improves for increasing $N$. As the constants in our theoretical results may depend on the polynomial degrees, this good behaviour in practice could not be predicted, but suggests that those constants depend only moderately on $p$.

An $h$-adaptive algorithm was compared to a uniform mesh-refinement strategy in our last experiment. Algorithms $\left(A_{0}^{0,(0,0)}\right),\left(A_{0}^{1,(0,0)}\right),\left(A_{1 / 2}^{0,(0,0)}\right)$, and $\left(A_{1 / 2}^{1,(0,0)}\right)$ generate results displayed in Figure [7] The adaptive meshes refine towards the singular point seen on the bottom plot of Figure 7 This improved the experimental convergence rate from $2 / 3$ to 3 .

Remark 5.3. The numerical experiments allow the subsequent conclusions:

(i) Our overall experience with the error estimator $\eta_{N}$ is that it serves as an efficient and reliable error indicator provided the exact solution is smooth enough. 

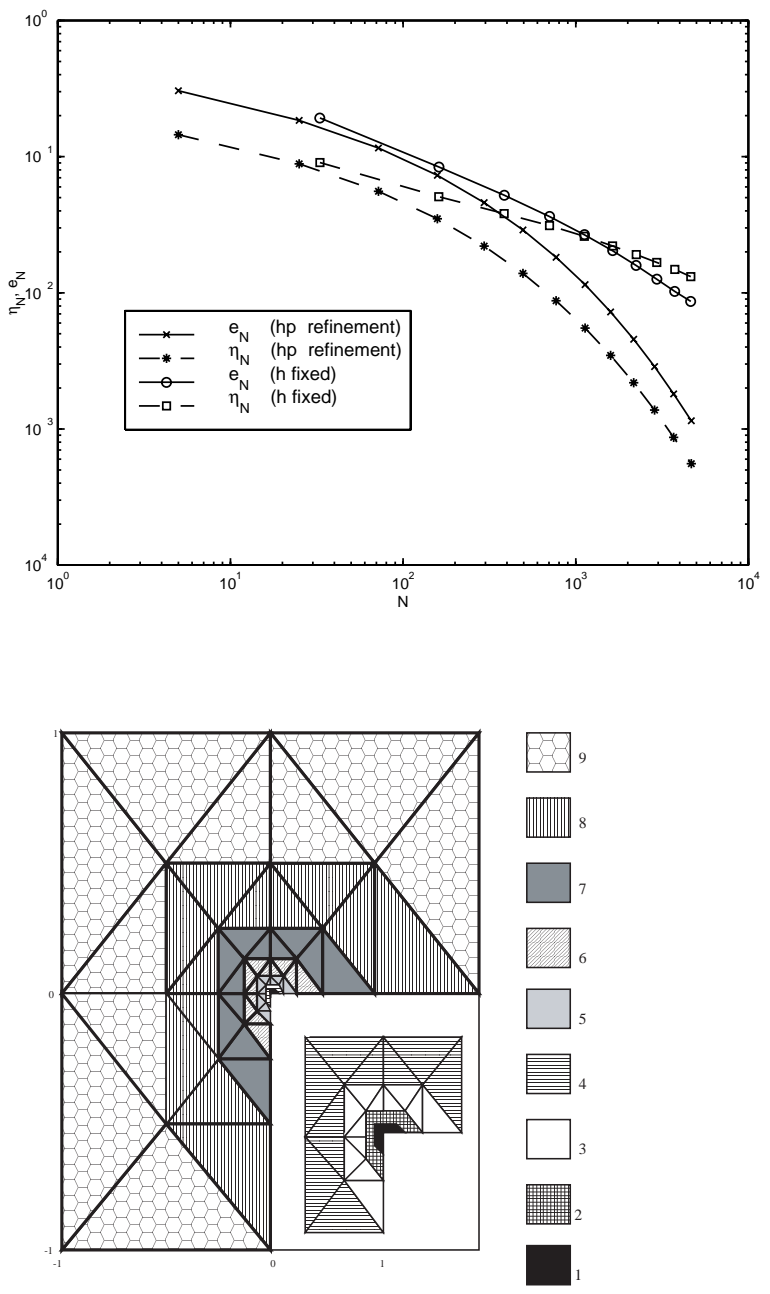

FiguRE 6. Error and error estimator for $h p$-adaptive meshrefinement strategy in Example 5.3 and for uniform p-refinement on the twice uniformly refined mesh $\mathcal{T}_{0}$ (top). Distribution of the polynomial degrees on $\mathcal{T}_{9}$ and magnified re-entrant corner (bottom).

For nonsmooth exact solutions there is no equivalence for high polynomial degrees in general, but $\eta_{N}$ is still reliable.

(ii) For smooth exact solutions, the constant in the efficiency estimate tends to one as the polynomial degree is increased. This behaviour is in agreement with Formula (4.27) of Theorem 4.3

(iii) The numerical experiments show that adaptive mesh-refinement strategies of Algorithm $\left(A_{\Theta}^{\vartheta, \delta}\right)$ yield considerable convergence improvements.

(iv) As is argued in [CB], the numerical examples support the theoretical prediction that the higher order terms in the reliability estimate depend on the smoothness of given right-hand sides, while those in the efficiency estimate depend on the smoothness of the exact solution. 

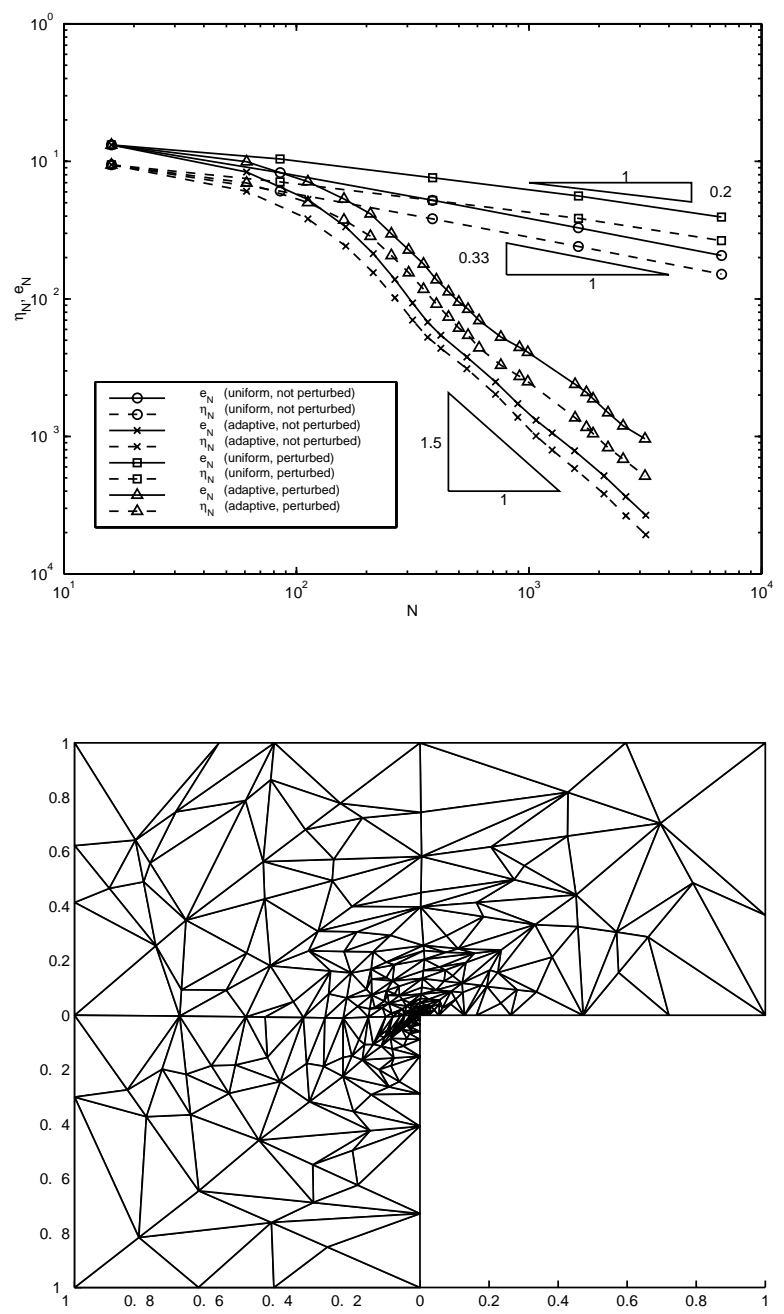

FIGURE 7. Error and error estimator for uniform and adaptive mesh-refinement on perturbed and unperturbed meshes in Example 5.3 (top) for $p=3$. Triangulation $\mathcal{T}_{22}$ with 382 nodes obtained from Algorithm $\left(A_{1 / 2}^{1,(0,0)}\right)$ (bottom).

Acknowledgments. The first author (S.B.) thankfully acknowledges partial support by the German Research Foundation (DFG) within the Graduiertenkolleg "Effiziente Algorithmen und Mehrskalenmethoden".

\section{REFERENCES}

[ACF] J. Alberty, C. Carstensen, S.A. Funken: Remarks around 50 lines of Matlab: short finite element implementation. Numer. Algorithms 20 (1999) 117-137. CMP 2000:01

[Baetal] I. BabušKa, T. Strouboulis, C.S. Upadhyay, S.K. GangaraJ, K. Copps: Validation of a posteriori error estimators by numerical approach. Int. J. Numer. Meth. Engrg. 37 (1994) 1073-1123. MR 95e:65096 
[BeR] R. Becker, R. RAnNACher: A feed-back approach to error control in finite element methods: basic analysis and examples. East-West J. Numer. Math., 4, No. 4 (1996) 237-264. MR 98m:65185

[B] D. Braess: Finite Elements. Cambridge University Press (1997). MR 98f:65002

[BV] D. Braess, R. Verfürth: A posteriori error estimators for the Raviart-Thomas element. SIAM J. Numer. Anal. 33 (1996) 2431-2444. MR 97m:65201

[BS] S.C. Brenner, L.R. ScotT: The Mathematical Theory of Finite Element Methods. Texts Appl. Math. 15, Springer, New-York (1994). MR 95f:65001

[Ca] C. Carstensen: Quasi interpolation and a posteriori error analysis in finite element method, M2AN 33 (1999) 1187-1202.

[CB] C. CARstensen, S. BARtels: Each averaging technique yields reliable a posteriori error control in FEM on unstructured grids. Part I: Low order conforming, nonconforming, and mixed FEM, Math. Comp., posted on February 4, 2002, PII S 0025-5718(02)014023 (to appear in print).

[CF] C. Carstensen, S.A. Funken: Constants in Clément-interpolation error and residual based a posteriori error estimates in Finite Element Methods. East-West J. Numer. Anal. 8 (2000), 153-175. CMP 2001:07

[CMS] C. Carstensen, S. Maischak, E.P. Stephan: A posteriori error estimate and $h$ adaptive algorithm on surfaces for Symm's integral equation. Numer. Math. (2001) Published online May 30, 2001, DOI 10.1007/s002110100287.

[CV] C. CARstensen, R. Verfürth: Edge residuals dominate a posteriori error estimates for low order finite element methods. SIAM J. Numer. Anal. 36 No. 5 (1999) 1571-1587. MR 2000g:65125

[Cl] P. ClÉment: Approximation by finite element functions using local regularization. RAIRO Sér. Rouge Anal. Numér. R-2 (1975) 77-84. MR 53:4569

[Ci] P.G. Ciarlet: The finite element method for elliptic problems. North-Holland, Amsterdam (1978). MR 58:25001

[EEHJ] K. Eriksson, D. Estep, P. Hansbo, C. Johnson: Introduction to adaptive methods for differential equations. Acta Numerica (1995) 105-158. MR 96k:65057

[GR] V. Girault, P.A. Raviart: Finite Element Methods for Navier-Stokes Equations. Springer, Berlin (1986). MR 88b:65129

[MBa] J.M. Melenk, I. BabušKa: The partition of unity finite element method: Basic theory and applications. Comput. Methods Appl. Mech. Eng. 139, No.1-4, 289-314 (1996). MR 97k:65258

[R] R. Rodriguez: Some remarks on Zienkiewicz-Zhu estimator. Int. J. Numer. Meth. in PDE 10 (1994) 625-635. MR 95e:65103

[V] R. VERFüRTH: A review of a posteriori error estimation and adaptive mesh-refinement techniques. Wiley-Teubner (1996).

[Y1] D. YU: Asymptotically exact a posteriori error estimators for elements of bi-odd degree. Chinese J. Num. Math. and Appl. 13, no. 4, 82-90 (1991). MR 94h:65103

[Y2] D. YU: Asymptotically exact a posteriori error estimators for elements of bi-even degree. Chinese J. Num. Math. and Appl. 13, no. 2, 64-78 (1991). MR 92i:65173

[ZZ] O.C. ZIENKIEWICZ, J.Z. ZHU: A simple error estimator and adaptive procedure for practical engineering analysis. Int. J. Numer. Meth. Engrg. 24 (1987) 337-357. MR 87m:73055

Mathematisches Seminar, Christian-Albrechts-Universität ZU Kiel Ludewig-MeynStr. 4, D-24098 KIEL, GERMANY

E-mail address: sba@numerik.uni-kiel.de

Institute for Applied Mathematics And Numerical AnAlysis, Vienna University of Technology, Wiedner Hauptstrasse 8-10, A-1040 Vienna, Austria

E-mail address: Carsten.Carstensen@tuwien.ac.at 